\title{
Region Clustering Based Evaluation of Multiple Top- $N$ Selection Queries
}

\author{
Liang Zhu ${ }^{\mathrm{a}, \mathrm{b}}$, Weiyi Meng ${ }^{\mathrm{c}, *}$, Wenzhu Yang ${ }^{\mathrm{a}}$, Chunnian Liu ${ }^{\mathrm{b}}$ \\ ${ }^{a}$ School of Mathematics and Computer Science, Hebei University, Baoding, Hebei 071002, China \\ ${ }^{\mathrm{b}}$ College of Computer Science and Technology, Beijing University of Technology, Beijing 100022, China \\ ${ }^{c}$ Department of Computer Science, State University of New York at Binghamton, Binghamton, NY 13902, USA
}

\begin{abstract}
In many database applications, there are opportunities for multiple top- $N$ queries to be evaluated at the same time. Often it is more cost effective to evaluate multiple such queries collectively than individually. In this paper, we propose a new method for evaluating multiple top- $N$ queries concurrently over a relational database. The basic idea of this method is region clustering that groups the search regions of individual top- $N$ queries into larger regions and retrieves the tuples from the larger regions. This method avoids having the same region accessed multiple times and reduces the number of random I/O accesses to the underlying databases. Extensive experiments are carried out to measure the performance of this new strategy and the results indicate that it is significantly better than the naïve method of evaluating these queries one by one for both low-dimensional $(2,3$, and 4$)$ and high-dimensional $(25,50$, and 104$)$ data.
\end{abstract}

Keywords: Top- $N$ Query; Multiple Queries Evaluation; Region Clustering

\section{INTRODUCTION}

Researches on top- $N$ selection queries have intensified in recent years (since late 1990s). Finding efficient strategies to evaluate top- $N$ queries has been one of the primary focuses of top- $N$ query research and has received much attention $[3,5,6,7,11,12,13,25,37,39,41,43]$. Most current work on top- $N$ query evaluation considers one query at a time. But there are many applications where multiple top- $N$ queries are available for processing. For example, a popular website may receive multiple top- $N$ queries (say finding the best used cars based on various conditions) at about the same time. As another example, a headhunting company (website) may have many job

\footnotetext{
* Corresponding author.

E-mail addresses: zhu@mail.hbu.edu.cn (L.Zhu), meng@cs.binghamton.edu (W. Meng), wenzhuyang@mail.hbu.edu.cn (W. Yang), ai@bjut.edu.cn (C. Liu)
} 
openings and may receive many resumes for these openings, and the company wants to find the top- $N$ matches (the best matching resumes) for every job opening [26]. In these and other similar situations, one can either employ an existing technique to evaluate the top- $N$ queries one by one or use an algorithm to evaluate these queries collectively. In this paper, we propose a new method to evaluate multiple top- $N$ queries collectively. We show that this method is significantly more efficient than the one-query-at-a-time method.

Example 1. Consider a database of used cars. Let the schema of the used cars be: Usedcars(id\#, make, model, year, price, mileage). Many prospective used car buyers may submit their queries with conditions on year, price, and mileage concurrently, and want to find the top 20 matches to each of these queries. Naturally, how to respond to these prospective buyers as quickly as possible is a problem that needs a solution.

A top- $N$ query may be evaluated in three steps. (1) A search range is determined based on some technique $[5,11$, 43] for each attribute in the query condition. If $n$ attributes are involved, then these ranges form an $n$-dimensional hyper rectangle (or region). (2) The tuples (data points) in the search region are retrieved. (3) The retrieved tuples are ranked based on their distances with the query and the top $N$ tuples are displayed.

When evaluating multiple top- $N$ queries, one way is to evaluate them one by one independently, we call this method the Naïve Method (NM) in this paper. Another method is to analyze the relationships among the search regions of these queries, group those that are close to each other (say having large overlaps) into clusters and then evaluate the queries in each cluster collectively. This is the main idea of our Region Clustering Method (RCM) to be presented in this paper. When a cluster has multiple regions, we find the smallest containing region $(S C R)$ that contains all these regions, and retrieve all the tuples from this $S C R$ into memory; then we identify the tuples for each individual region, compute their distances with respect to their queries to find the top- $N$ tuples for each query. Intuitively, if many regions in a cluster are close to each other and have overlaps, RCM may be more efficient for the following reasons. First, the number of random I/Os can be reduced. Retrieving tuples from each region incurs at least one random I/O and when there are many regions, many random I/Os will occur. In contrast, when a (larger) continuous space (the $S C R$ ) is searched, most I/Os are likely to be sequential I/Os. As random I/Os are much more expensive than sequential I/Os, RCM is likely to yield lower I/O cost. Second, the possibility of retrieving the same tuple multiple times is avoided. If a tuple appears in $k$ different regions, then the tuple will be retrieved $k$ times using NM, but only once by RCM if the $k$ regions are clustered together. On the other hand, RCM may retrieve more tuples that are not needed by any query as the $S C R$ may cover areas that are not covered by any of the regions. 
Therefore, for RCM to be effective, the clustering must be carried out in such a way that maximizes the benefits while minimizing the loss. The main focus of this paper is to develop effective region clustering strategies. We assume that the search region for each top- $N$ query has been obtained using an existing method $[5,11,43]$.

In the literature, the phrase "multiple top- $N$ queries" has been used in different contexts with different meanings. In [17], it means to find the top- $N$ results that are closest to a set of queries based on a collective distance measure. This type of queries is also known as group nearest neighbour queries [31] or multipoint queries [8]. In [29], multiple scoring functions are defined for the same query and "multiple top- $N$ queries" means to find a set of top- $N$ results with respect to each scoring function for the same query at the same time. In this paper, we consider the simultaneous evaluation of multiple independent top- $N$ queries. Note that for the most part our approach is independent of the scoring function(s) used because the input to our approach is a set of search regions of individual top- $N$ queries; it does not matter to us if these regions are computed based on different scoring functions. Thus, the kind of multiple top- $N$ queries we consider in this paper are different from those considered in previous work. More details about the differences will be provided in Section 2.

The main contribution of this paper is the development and evaluation of a region clustering based method for evaluating multiple top- $N$ queries collectively. Note that even though this method is presented in the context of evaluating multiple top- $N$ queries, it is directly applicable to evaluating multiple range queries in multi-dimensional spaces. This method can be used in arbitrary dimensional spaces and it works well for both low-dimensional and high-dimensional data. In our experiments, datasets with 2, 3 and 4 dimensions (low dimensions) and 25, 50 and 104 (high dimensions) are used. Our experimental results indicate that this method can lead to significant cost savings over the naïve one-query-at-a-time method. To the best of our knowledge, the problem of evaluating the kind of multiple top- $N$ queries that we consider in this paper has not been investigated before.

The rest of the paper is organized as follows. In Section 2, we briefly review some related works. In Section 3, we introduce some notations and provide a brief analysis on the cost of evaluating multiple top- $N$ queries. In Section 4, we present our Region Clustering Method (RCM) to top- $N$ query evaluation. In Section 5, we present the experimental results. Finally, in Section 6 we conclude the paper.

\section{RELATED WORK}

The need to rank the results of database queries has long been recognized. Motro [28] gave the definitions of vague queries. He emphasized the need to support approximate and ranked matches in a database query language, and 
introduced vague predicates. Carey and Kossmann $[6,7]$ proposed techniques to optimize top- $N$ queries when the scoring is done through the SQL "Order By" clause by reducing the size of intermediate results. Onion [9] and Prefer $[18,19]$ are preprocessing-based techniques for top- $N$ queries. For a linear preference function, the basic observation of Onion is that the tuples with the highest score can be found within the convex hull of the dataset. Prefer considers both linear [18] and non-linear [19] scoring functions, provided that a different set of views is maintained for each function type. In addition, a variety of algorithms for materialized top- $N$ views have been proposed in $[12,40]$. The authors of [13] use a probabilistic approach to optimize top- $N$ queries. The ranking condition in [13] involves only a single attribute. [39] proposed a computational model, ranking cube, for efficient answering of top- $N$ queries with multidimensional selections.

Fagin et al. [15] introduced the threshold algorithms (TA) that perform index scans over pre-computed index lists, one for each attribute or keyword in the query, which are sorted in descending order of per-attribute or perkeyword scores. Variations of TA have been proposed for several applications, including similarity search in multimedia repositories [10], approximate top- $N$ retrieval with probabilistic guarantees [37], scheduling methods based on a Knapsack-related optimization for sequential accesses and a cost model for random accesses [3], and the distributed TA-style algorithm has been presented in [25, 27].

The query model for top- $N$ queries in $[5,11,43]$ is consistent with the definitions in [28]. In $[5,11,43]$, the key focus is on exploring the strategies that map a top- $N$ query into a traditional range selection query. The basic idea of these strategies is to find an appropriate search region centered at the query point of any given top- $N$ query such that all of the desired tuples (i.e. the top- $N$ tuples) but very few undesired ones are contained in the search region.

Most existing works focus on the evaluation of top- $N$ queries with only selection conditions (no joins). In this paper, we present a method to evaluate multiple such selection queries efficiently. This paper does not focus on how to find the best search region for each top- $N$ query, instead the search regions of all input queries are used as input to our region clustering method. In other words, our method relies on existing techniques to find the search regions for individual top- $N$ queries. Specifically, we can say that the work to be reported in this paper is built on top of the techniques described in $[5,11,43]$. Since the histogram-based approaches in [5] are not suitable for high dimensional data sets $[5,23]$ and the sampling-based method in [11] only provides an approximate answer to a given top- $N$ query (i.e., it does not guarantee the retrieval of all the top- $N$ tuples), we use the method described in [43] to provide the search region for individual queries in this paper. The learning-based method in [43] can learn from 
either randomly generated training queries or real user queries. Furthermore, it delivers good performance for either low-dimensional data or high-dimensional data and guarantees the retrieval of all top- $N$ tuples for each query.

$\mathrm{Yu}$ et al. [41, 42] introduced the methods for processing top- $N$ queries in multiple database environments. The techniques are based on ranking databases. [42] uses histograms and [41] considers the information of past queries in top- $N$ query evaluation. The authors of [2] developed methods to optimize the communication costs in P2P networks. In this paper, our environment consists of a single database at a central location.

Top- $N$ queries involving joins have also been considered [6, 17, 20, 21, 22, 24, 44]. Ilyas et al. [20] introduced a pipelined rank-join operator, based on the ripple join technique. [21] proposed an algorithm that is suitable for evaluating a hierarchy of join operators. The RankSQL work $[22,24]$ considered the order of binary rank joins at query-planning time. For the planning time optimization, RankSQL uses simple statistical models, assuming that scores within a list follow a normal distribution [22]. We consider only selection queries in this paper and join queries will be considered in the future.

Multiple-query processing was first studied in late 1980s [32,33], and it still is an active research area [1, 14, $35,36,38]$. But existing works do not consider multiple top- $N$ queries. The authors of [16] proposed a UB-tree based method to process a set of query boxes (i.e., range queries), then used query boxes to approximate a nonrectangular shape (say, a triangle). The goal of this work was to minimize the number of loaded pages that overlap and the experiments were based on 2-dimensional data. As mentioned in [4], index-based method is efficient for databases with a small number of attributes, but not suitable for high-dimensional space (larger than 12). Our Region Clustering Method (RCM) does not use any index and is suitable for high-dimensional dataset (104 dimensions in our experiments).

The phrase "multiple top- $N$ queries" has appeared in several recent papers but it does not have the same meaning as the type of multiple top- $N$ queries we consider in this paper. In [17], it means to find the top- $N$ results that are closest to a set of queries based on a collective distance measure. This type of queries is also known as group nearest neighbour (GNN) queries [31] or multipoint queries [8]. As an example, in [31], the problem is to find the $N(\geq 1)$ data point(s) with the smallest sum of distances to all query points in a given query set $Q=\left\{Q_{1}, \ldots, Q_{m}\right\}$. Clearly, this problem is very different from the problem we are trying to solve in this paper, namely, find the top- $N$ tuples for each query of a given set of queries. The example in Figure 1(a) can be used to illustrate the difference. In Figure 1(a), $\left\{P_{1}, P_{2}, P_{3}\right\}$ are three data points and $\left\{Q_{1}, Q_{2}\right\}$ are two query points in a 2-dimensional Euclidean space. 
Based on the distances given in the figure, $P_{3}$ is the desired data point for the GNN problem with $N=1$, because $d\left(\left\{Q_{1}, Q_{2}\right\}, P_{3}\right)=d\left(Q_{1}, P_{3}\right)+d\left(Q_{2}, P_{3}\right)=4$ is the minimum value in $\left\{d\left(\left\{Q_{1}, Q_{2}\right\}, P_{i}\right), i=1,2,3\right\}$. For our problem, we need to find the top- $N$ results for each query; when $N=1$, the top-1 tuples for $Q_{1}$ and $Q_{2}$ are $P_{1}$ and $P_{2}$, respectively. In [8], the sum of weighted distances is used instead of the sum of distance in [31]. The collective distance measure used in [17] is the minimum of distances, i.e., $d(P, Q)=\min _{N}\left\{d\left(P, Q_{i}\right) \mid Q_{i} \in Q, i=1, \ldots, m\right\}$ is used to define the top- $N$ result tuple(s). In Figure 1(b), for instance, the set of multiple query points is $Q=\left\{Q_{1}, Q_{2}, Q_{3}\right\}$ and the three tuples are $P_{1}, P_{2}$ and $P_{3}$. The top-1 tuple of the set $\mathrm{Q}=\left\{Q_{1}, Q_{2}, Q_{3}\right\}$ is $P_{3}$ according to the definitions in [17]; however, in our paper, the top-1 tuples for $Q_{1}, Q_{2}$ and $Q_{3}$ are $P_{1}, P_{2}$ and $P_{3}$ respectively.

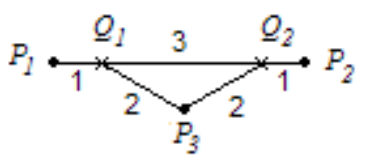

(a)

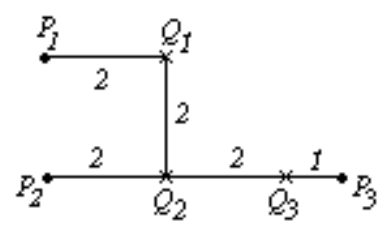

(b)

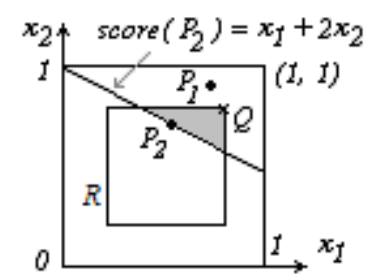

(c)

Fig.1. Illustrations of the differences of top- $N$ queries

The authors of [29] studied continuous monitoring of top- $N$ queries over a fixed-size window of the most recent data. This paper also discussed the processing of "multiple top- $N$ queries". However, there are two major differences between the work in [29] and our work reported in this paper. First, in [29], the query point is always at the upperright corner of the restricted region (see region $R$ in Figure 1(c) for a 2-dimensional example) and only top- $N$ results in a specific area (see the grey triangle in Figure 1(c)) are considered. Our work does not have these restrictions. Second, in [29], only one query point is considered at a time but multiple scoring functions are defined for the same query; the objective is to find a set of top- $N$ results with respect to each scoring function for the same query at the same time. In contrast, our work considers multiple query points and tries to find the top- $N$ results for each query.

\section{PROBLEM DEFINITION AND ANALYSIS}

Let $\mathfrak{R}^{n}$ be an $n$-dimensional metric space with distance function $d\left(\right.$ ), where $\mathfrak{R}$ is the real line. Suppose $\boldsymbol{R} \subset \mathfrak{R}^{n}$ is a relation (or dataset) with $n$ attributes $\left(A_{1}, \ldots, A_{n}\right)$. Consider a query point $Q=\left(q_{1}, \ldots, q_{n}\right) \in \mathfrak{R}^{n}$ and an integer $N>0$. A top- $N$ selection query $(Q, N)$, or top- $N$ query for short, is to find a sorted set of $N$ tuples in $\boldsymbol{R}$ that are closest to $Q$ according to the given distance function. The results of a top- $N$ query are called top- $N$ tuples. We assume $N<|\boldsymbol{R}|$ (the size of $\boldsymbol{R}$ ); otherwise, we just retrieve all tuples in $\boldsymbol{R}$. 
As mentioned in Section 1, a number of approaches exist for mapping a top- $N$ query on a relational database into a traditional range selection query. Specifically, for a given top- $N$ query $(Q, N)$, these approaches can determine a search region, denoted $R(Q, N)$, for the top- $N$ query.

In this paper, a region $R=\prod_{i=1}^{n}\left[a_{i}, b_{i}\right]$ is always an $n$-dimensional hyper rectangle, and we use $v(R)=\prod_{i=1}^{n}\left(b_{i}-\right.$ $\left.a_{i}\right)$ to denote the volume of $R$. Let $\left\{Q_{1}, \ldots, Q_{m}\right\} \subset \mathfrak{R}^{n}$ be a set of $m$ points, and $N_{l}, \ldots, N_{m}$ be $m$ positive integers, $m>$ 1. In this paper, we discuss how to efficiently evaluate the set of $m$ top- $N$ queries, $Q=\left\{\left(Q_{1}, N_{l}\right), \ldots,\left(Q_{m}, N_{m}\right)\right\}$.

We now use an example to explain the differences in evaluation costs between NM and RCM. Figure 2 shows the search regions $R\left(Q_{1}, N_{l}\right)$ and $R\left(Q_{2}, N_{2}\right)$ of two top- $N$ queries $\left(Q_{l}, N_{l}\right)$ and $\left(Q_{2}, N_{2}\right)$ in $\Re^{2}$, respectively. The smallest containing region $(S C R)$ of these two regions is the rectangle with the dotted line.

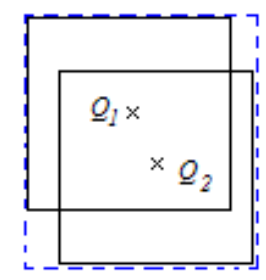

Fig.2. The $S C R$ and search regions of $Q_{1}$ and $Q_{2}$

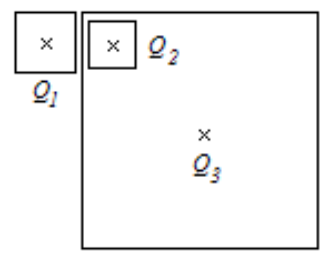

Fig.3. Clustering of three top- $N$ queries

As explained in Section 1, a typical top- $N$ query can be processed in three steps. Let $R C($ ) denote the cost of constructing the search region, $I O C($ ) the cost of evaluating the query (mostly $\mathrm{I} / \mathrm{O}$ cost), and $S C($ ) the cost of sorting and displaying the results. Using NM, the total cost for evaluating $\left(Q_{1}, N_{l}\right)$ and $\left(Q_{2}, N_{2}\right)$ is as follows:

$$
\text { Cost_NM }=R C\left(Q_{1}, N_{l}\right)+\operatorname{IOC}\left(Q_{1}, N_{l}\right)+S C\left(Q_{1}, N_{l}\right)+R C\left(Q_{2}, N_{2}\right)+\operatorname{IOC}\left(Q_{2}, N_{2}\right)+S C\left(Q_{2}, N_{2}\right)
$$

When RCM is used, we still need to construct the search regions for the two queries as in NM. Then we construct the $S C R$ and retrieve all tuples from $S C R$ into the memory. Next, we identify the retrieved tuples that belong to $R\left(Q_{1}, N_{I}\right)$ and $R\left(Q_{2}, N_{2}\right)$, respectively. Finally, sort the tuples in $R\left(Q_{1}, N_{1}\right)$ and $R\left(Q_{2}, N_{2}\right)$, and output the top- $N$ tuples for $Q_{1}$ and $Q_{2}$, respectively. Thus, using RCM, the total cost will be:

$$
\text { Cost_RCM }=R C\left(Q_{1}, N_{l}\right)+R C\left(Q_{2}, N_{2}\right)+I O C(S C R)+S C\left(Q_{1}, N_{l}\right)+S C\left(Q_{2}, N_{2}\right)+\delta
$$

where $\delta$ denotes all the extra cost (including the cost for constructing the $S C R$, performing the clustering and identifying the retrieved tuples that belong to each individual search region). In Expression (1), there are two independent $\mathrm{I} / \mathrm{O}$ requests and in Expression (2), there is only one $\mathrm{I} / \mathrm{O}$ request. Suppose each $\mathrm{I} / \mathrm{O}$ request incurs one random I/O plus some sequential I/Os. Since the cost of a random I/O is about 10 times higher than that of a 
sequential I/O [30], query evaluation strategies that incur lower random I/Os are likely to be more efficient. As I/O cost is usually the main cost in most database applications, reducing the $\mathrm{I} / \mathrm{O}$ cost, especially the random $\mathrm{I} / \mathrm{O}$ cost, is an effective way to improve the efficiency of query evaluation.

The RCM we propose in this paper aims to identify situations where the total cost for RCM is smaller than the total cost for NM. The reason we employ region clustering rather than (query) point clustering is because top- $N$ queries are better characterized by their search regions instead of just the query points. Consider the example in Figure 3. Since $R\left(Q_{3}, N_{3}\right) \supset R\left(Q_{2}, N_{2}\right), Q_{3}$ and $Q_{2}$ should be in the same cluster although the distance $d\left(Q_{1}, Q_{2}\right)$ is less than $d\left(Q_{3}, Q_{2}\right)$. In this case, if we have retrieved all the tuples in $R\left(Q_{3}, N_{3}\right)$ from a database, we can obtain the top- $N$ tuples for $Q_{2}$ from those in $R\left(Q_{3}, N_{3}\right)$ without additional I/Os. If we place $Q_{1}$ and $Q_{2}$ in the same cluster, the above savings on I/Os cannot be obtained.

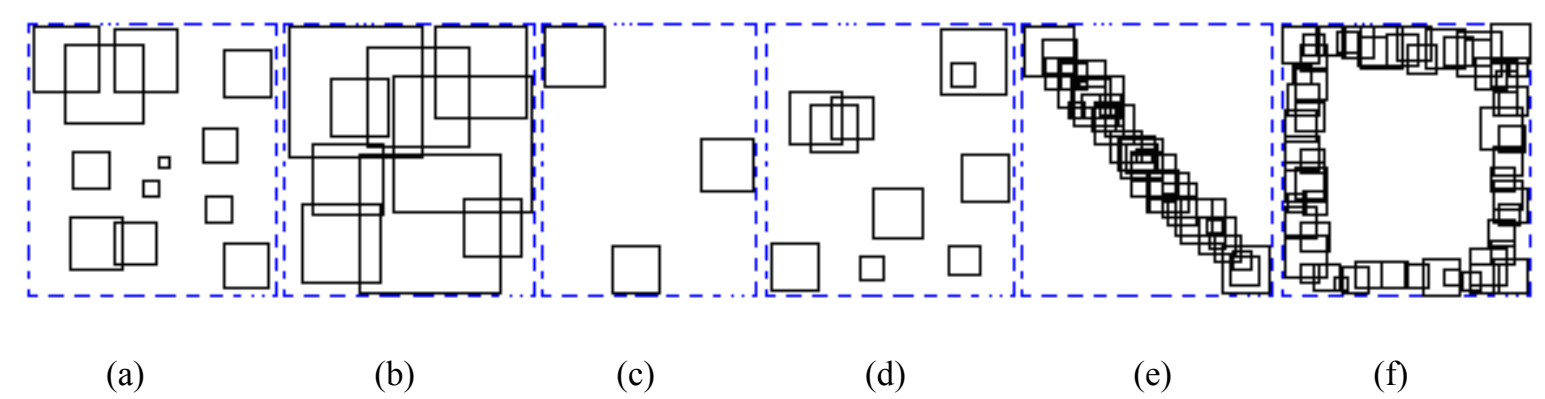

Fig. 4. Examples of the distributions of regions

Clearly, the distribution of the search regions of the input queries plays an important role in how to evaluate these queries collectively. We now provide some measures to characterize the distribution of a set of regions. Figure 4 shows several examples of the distributions of some 2-dimensional regions. Let $R_{l}, R_{2}, \ldots, R_{k}$ be $k$ regions. By comparing the three volumes $\sum_{i=1}^{k} v\left(R_{i}\right), v\left(\cup_{i=1}^{k} R_{i}\right)$ and $v\left(S C R\left(\cup_{i=1}^{k} R_{i}\right)\right)$, we can gain useful insight about the distribution of these regions. Denote

$$
\alpha=v\left(\bigcup_{i=1}^{k} R_{i}\right) / \sum_{i=1}^{k} v\left(R_{i}\right), \beta=v\left(\bigcup_{i}^{k} R_{i}\right) / v\left(\operatorname{SCR}\left(\bigcup_{i=1}^{k} R_{i}\right)\right), \tau=v\left(\operatorname{SCR}\left(\bigcup_{i=1}^{k} R_{i}\right)\right) / \sum_{i=1}^{k} v\left(R_{i}\right) .
$$

It is easy to see that $\alpha \leq 1$ and $\beta \leq 1$. The values of $\alpha$ indicate the degree of overlap of the regions and a smaller $\alpha$ indicates more overlap. The values of $\beta$ reflect the closeness between the regions and the size of the lacuna in the $S C R$; a larger $\beta$ indicates closer regions and smaller lacuna in general. The value of $\tau$ may be greater than, less than or equal to 1 , and it indicates how much of the $S C R$ is occupied by the $R_{i}$ 's; a smaller $\tau$ denotes larger volumes of $R_{i}$ 's in the $S C R$. If $\tau<1$, then there must be some $R_{i}$ 's that overlap one another. 
In some cases, using the $S C R$ of $\cup_{i=1}^{k} R_{i}$ as a single search region for all $R_{1}, R_{2}, \ldots, R_{k}$ may be an effective strategy like in the case shown in Figure 4(b), which has "good measures", that is, a small $\alpha$, a large $\beta$ and a small $\tau$. However, in other cases, it may not be appropriate to form just one cluster as it may lead to the retrieval of many useless tuples, like in the cases shown in Figure 4(e)-(f), which have "good $\alpha$ and $\tau$ " (small $\alpha$ and $\tau$ ), but a "bad $\beta$ " (small $\beta$ ). Intuitively, it seems to be more appropriate to form several smaller clusters for the regions along the diagonal in Figure 4(e) and 4 clusters for the regions in Figure 4(f) along the 4 edges. Note that if clusters were formed simply based on overlapping regions, then all the regions in Figure 4(e) and (f) would form a single cluster, and such SCRs would contain too many useless tuples. Figure 4 (a), (c) and (d) represent more random cases and they all have "bad measures", that is, a large $\alpha$, a small $\beta$ and a large $\tau$. Figure 4(c) is the worst case with $\alpha=1$. Therefore, the three regions should not be grouped into a single cluster; in other words, they should be evaluated individually, i.e., each region is considered as a separate cluster. Figure 4(a) and (d) are similar in that in each case some regions overlap and some don't, and $\alpha<1$. For the regions in Figure 4(d), it is intuitively reasonable for the overlapping regions to form two clusters and the remaining regions to remain un-clustered. However, it is less clear how the regions in Figure 4 (a) should be clustered. A general algorithm for region clustering will be presented in Section 4.3.

\section{MULTIPLE TOP-N QUERY PROCESSING}

The key step of our approach is to group the search regions of $m$ input top- $N$ queries into clusters. All current clustering techniques employ some similarity measure to compute the distance among the objects to be clustered. In most cases, the objects are points. However, the objects are regions in this paper.

\subsection{Algorithms and Terminologies}

In this section, we introduce the basic algorithms and terminologies that will be used by RCM. The algorithms include: (1) VUR, that computes the volume of the union of multiple regions; (2) DTR, that calculates the difference of two regions; (3) PPS, that partitions multiple points in $n$-dimensional space; (4) CST, that gets the set of candidate top- $N$ tuples of a top- $N$ query; and (5) TTC, that gets the top- $N$ tuples from the set of candidate top- $N$ tuples. 


\subsubsection{The Volume of the Union of $m$ Regions (VUR)}

Given $m$ regions $R_{l}, R_{2}, \cdots, R_{m}, v\left(R_{i}\right)$ denotes the volume of $R_{i}$. Let $v_{1}=\sum_{1 \leq i \leq m} v\left(R_{i}\right), v_{2}=\sum_{1 \leq i<j \leq m} v\left(R_{i} \cap R_{j}\right), v_{3}=$ $\sum_{1 \leq i<j<k \leq m} v\left(R_{i} \cap R_{j} \cap R_{k}\right), \ldots$, and $v_{m}=v\left(R_{l} \cap R_{2} \cap \cdots \cap R_{m}\right)$, then by induction, we have

$$
v\left(\cup_{i=1}^{m} R_{i}\right)=v_{1}-v_{2}+v_{3}-v_{4}+\ldots+(-1)^{m-1} v_{m} .
$$

Suppose $R_{l}=\prod_{i=1}^{n}\left[a_{i}, b_{i}\right]$ and $R_{2}=\prod_{i=1}^{n}\left[c_{i}, d_{i}\right]$ are two $n$-dimensional regions. Their intersection $R_{l} \cap R_{2}$ is also an $n$-dimensional region. Let $p_{i}=\max \left\{a_{i}, c_{i}\right\}$, and $q_{\mathrm{i}}=\min \left\{b_{i}, d_{i}\right\}, i=1,2, \ldots, n$. If there is an $i_{0}$ such that $p_{i_{0}}>q_{i_{0}}$, then $R_{1} \cap R_{2}=\phi$, and $v\left(R_{1} \cap R_{2}\right)=0$; otherwise, if $p_{i} \leq q_{i}(i=1,2, \ldots, n)$, then $R_{I} \cap R_{2} \neq \phi, R_{1} \cap R_{2}=\prod_{i=1}^{n}\left[p_{i}, q_{i}\right]$, and

$$
v\left(R_{1} \cap R_{2}\right)=\prod_{i=1}^{n}\left(q_{i}-p_{i}\right)
$$

Note that $v\left(R_{1} \cap R_{2}\right)=\prod_{i=1}^{n}\left(q_{i}-p_{i}\right)=0$ if there is an $i_{0}$ such that $p_{i_{0}}=q_{i_{0}}$, although $R_{1} \cap R_{2} \neq \phi$.

Based on the associativity of " $\cap$ ", Formulas (3) and (4), the exact volume of $\cup_{i=1}^{m} R_{i}$ can be computed. However, Formula (3) has a complexity of $\mathrm{O}\left(2^{m}\right)$. In this paper, we deal with this problem by first partitioning the set of regions into smaller sets and then process each subset separately. Note that since $R \cap \phi=\phi$, many items in Formula (3) are empty. As a result, the number of non-empty items in Formula (3) is usually much smaller than $2^{m}$. For example, if $R_{i} \cap R_{j}=\phi$ or $v\left(R_{i} \cap R_{j}\right)=0,1 \leq i<j<m$, then $R_{i} \cap R_{j} \cap R_{k}=\phi$ or $v\left(R_{i} \cap R_{j} \cap R_{k}\right)=0$, for all $k, j<k \leq m$, and all such items can be discarded from Formula (3).

\subsubsection{The Difference of Two Regions (DTR)}

Let $S=\prod_{i=1}^{n}\left[a_{i}, b_{i}\right]$ and $T=\prod_{i=1}^{n}\left[c_{i}, d_{i}\right]$ be two regions. If $S \cap T \neq \phi$ and $T$ is not contained in $S$, then the difference $T$ - $S$ is the union of some $n$-dimensional sub-regions of $T$, that is, $T-S=\cup_{j=1}^{p} T_{j}$, where $T_{j} \subset T, j=1, \ldots, p, v\left(T_{i} \cap T_{j}\right)$ $=0, i \neq j$, can be constructed by the following algorithm: 


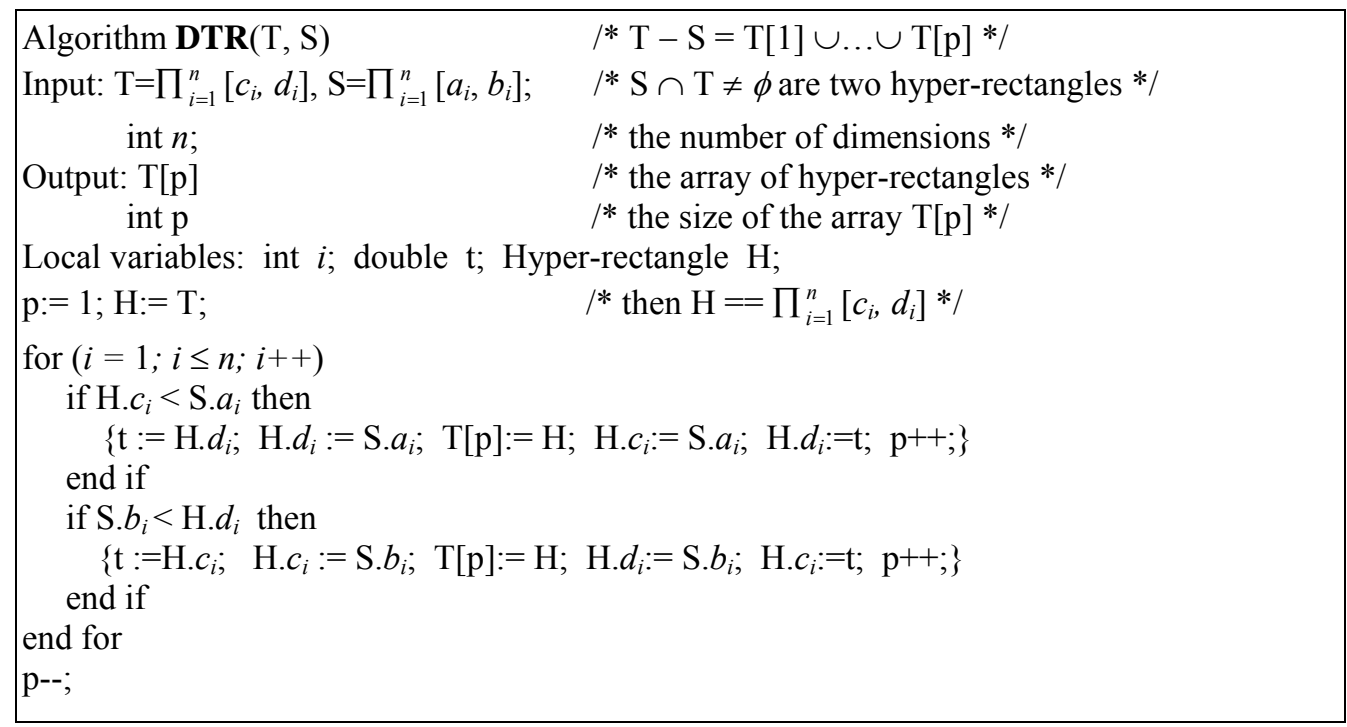

It is clear that Algorithm DTR also holds if $T$ is contained in $S$ and $T-S=\phi$. Figure 5 shows three cases of $T-S$ in 2-dimensional space.

We can use Algorithm DTR to reduce the query evaluation time in many cases. For example, if the tuples in $S$ in Figure 5(a) have been retrieved, it is sufficient to retrieve the tuples in $T_{1} \cup T_{2} \cup T_{3}$ and combine them with the tuples from $S$ that are in $T$ to obtain the tuples in $T$.

In general, the number of such $T_{j}$ 's (i.e., $\left.\left|\left\{T_{j}\right\}\right|\right)$ is not larger than $2 n$ in an $n$-dimensional space. In many cases, $\left|\left\{T_{j}\right\}\right|$ can be much smaller than $2 n$. For example, in Figures 5(b) and 5(c), $\left|\left\{T_{j}\right\}\right|$ is 2 and 1, respectively. Intuitively, DTR is effective only when $\left|\left\{T_{j}\right\}\right|$ is small because a large $\left|\left\{T_{j}\right\}\right|$ can lead to more expensive random I/Os. In our experiments, DTR is used only when $\left|\left\{T_{j}\right\}\right|$ is not larger than 2 .

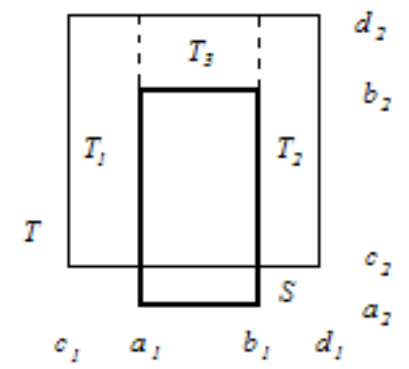

(a). $T-S=T_{1} \cup T_{2} \cup T_{3}$

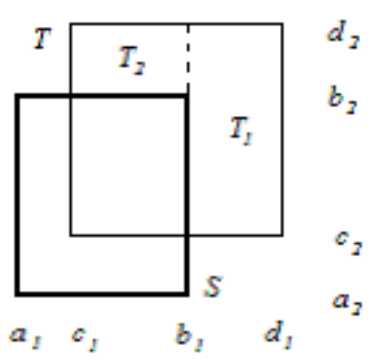

(b). $T-S=T_{1} \cup T_{2}$

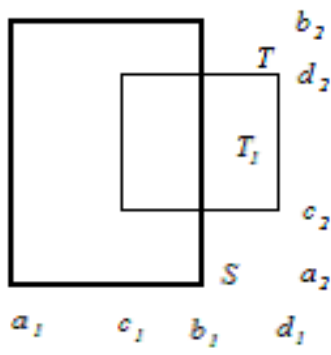

(c). $T-S=T_{1}$

Fig. 5. Three cases of $T-S$ in 2-Dimensional Space

\subsubsection{The Partition of Points in $n$-dimensional Space (PPS)}

Let $\mathrm{T}=\left\{t^{(l)}, \ldots, t^{(m)}\right\}$ be a set of $m$ points in $\mathfrak{R}^{n}$ and $R=\prod_{i=1}^{n}\left[a_{i}, b_{i}\right]$ be a region that contains these points. 
Given a threshold $m_{0}$, there is a number $p$ such that $R$ is divided into $p$ cells $\left\{P_{i}: i=1, \ldots, p\right\}$ and each cell $P_{i}$ contains at most $m_{0}$ points in $\mathrm{T}$. The following algorithm can achieve this:

Algorithm PPS(T, $\left.R, m_{0}, n_{1}, \ldots, n_{k}\right) / * n_{i}>1, i=1, \ldots, k * /$

(1) Find the $k$ longest edges of $R$, denote them as $e_{1}, e_{2}, \ldots, e_{k}$, and divide them into $n_{1}, n_{2}, \ldots, n_{k}$ equal-length segments respectively; then $R$ is partitioned into $h=n_{1} n_{2} \ldots n_{\mathrm{k}}$ cells $\left\{P_{i}: i=1, \ldots, h\right\}$.

(2) For each cell $P_{i}$ containing more than $m_{0}$ points in T, let $R:=P_{i}$ and goto (1).

It is clear that $p=h+\left(N_{i t e}-1\right) *(h-1)=h * N_{\text {ite }}-N_{\text {ite }}+1$, where $N_{\text {ite }}$ is the number of times step (1) is executed. It is easy to know the relationship between the depth $d$ of the partitions in PPS and the volume of the cells. Let $v_{(d)}$ denote the volume of a cell after $d$ consecutive partitions from $R$. Then $v_{(d)}=v(R) / h^{d}$. The convergence of the algorithm PPS is very fast, in fact, it is not slower than $\mathrm{O}\left(1 / 2^{d}\right)$, which is the slowest case with $k=1$ and $n_{1}=2$.

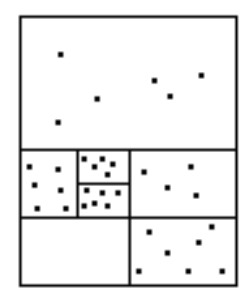

(a) $k=1, n_{1}=2$

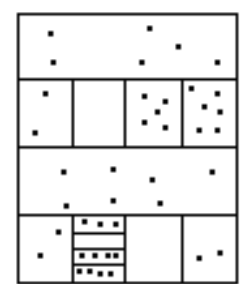

(b) $k=1, n_{1}=4$

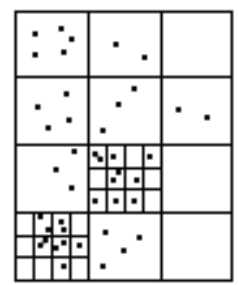

(c) $k=2, n_{1}=4, n_{2}=3$

Fig. 6. PPS in 2-Dimensional Space, $m_{0}=8$

Let $m_{0}=8$. Figures 6(a)-(c) illustrate three cases of the algorithm PPS in 2-dimensional space. Note that, in Figure 6(c), $R$ is divided into 3 segments along the $x$-axis and 4 segments along the $y$-axis; moreover, the two smaller cells have 4 segments along the $x$-axis and 3 segments along the $y$-axis.

In our experiments, threshold $m_{0}=8$ is used for all datasets of both low and high dimensions. The reason we choose $m_{0}=8$ is as follows. With $m_{0}=8$, each cell will have no more than 8 points. Each point corresponds to a top$N$ query, and as a result, it has a corresponding search region. This means that by considering the points in different cells separately, no more than 8 regions will be considered at a time. Note that the VUR algorithm has a complexity of $\mathrm{O}\left(2^{m}\right)$, where $m$ is the number of regions. When no more than 8 regions are considered at a time, the VUR algorithm can run very fast. For the 2 dimensional case, we take $k=2, n_{1}=4$, and $n_{2}=3$ as in Figure 6(c). For the $n$ dimensional case $(n \geq 3)$, we take $k=3, n_{1}=3, n_{2}=2$ and $n_{3}=2$. Thus, each time, the $S C R$ (or a cell) is partitioned into 12 smaller cells. For example, for the 104-dimensional dataset Lsi104D, we will find the 3 longest edges $e_{1}, e_{2}$ and $e_{3}$ of $S C R$ from all 104 edges in each partition. In the first partition, suppose $e_{1}, e_{2}$ and $e_{3}$ are the $5^{\text {th }}$, the $17^{\text {th }}$, and the $89^{\text {th }}$ dimensions, respectively, and by dividing them into 3,2, and 2 equal-length segments respectively, the $S C R$ 
is divided into $h=3 * 2 * 2=12$ cells $\left\{P_{i}: i=1, \ldots, 12\right\}$. In the next partition, for a cell, say $P_{3}, e_{1}, e_{2}$ and $e_{3}$ may belong to other dimensions.

\subsubsection{Get Candidate Set of Top- $N$ Tuples (CST)}

Let $(Q, N)$ be a top- $N$ query, and $R$ be its search region. If a tuple $t \in R$, it is said to be a candidate of the top- $N$ tuples of $(Q, N)$ (or candidate for short). The set of all candidates is called the candidate set of $(Q, N)$.

Given two regions $S=\prod_{i=1}^{n}\left[a_{i}, b_{i}\right]$ and $T=\prod_{i=1}^{n}\left[c_{i}, d_{i}\right]$, if the candidate set of $S$ has been obtained, then we can get the candidate set of $T$ using the following algorithm:

$\operatorname{Algorithm~} \operatorname{CST}(T, S)$

(1) if $T \subset S$, then the candidate set of $T$ is already obtained; simply identify the tuples from $S$ that are in $T:\{t$ : $t=\left(t_{1}, \ldots, t_{n}\right) \in S$ and $\left.c_{i} \leq t_{i} \leq d_{i}\right\}$;

(2) if $T \not \subset S$ and $T \cap S \neq \phi$, then obtain $T-S=\cup_{j=1}^{p} T_{j}$ using $\operatorname{DTR}(T, S)$; if $p$ is less than a threshold, get tuples $\left\{t^{(i)}\right\}$ from $\cup_{j=1}^{p} T_{j}$ and the candidate set of $T$ is $\left\{t^{(i)}\right\} \cup\left\{t: t=\left(t_{1}, \ldots, t_{n}\right) \in S\right.$ and $\left.c_{i} \leq t_{i} \leq d_{i}\right\}$;

(3) if $T \cap S=\phi$ or $p$ is not less than a threshold, get tuples in $T$ from the database directly;

Now we introduce the concept of best-super-region (BSR). Suppose that $\left\{R_{i}: i=1, \ldots, m\right\}$ is a set of regions, $R_{k}$ is called the BSR of $R_{i}$ if $R_{i} \subset R_{k}$ and $v\left(R_{k}\right)=\min \left\{v\left(R_{j}\right): R_{i} \subset R_{j}, 1 \leq j \leq m\right.$ and $\left.i \neq j\right\}$, i.e., $R_{k}$ is the smallest region that contains $R_{i}$. The index $k$ of $R_{k}$ is called the best-super-region number (BSR number) of $R_{i}$, denoted as $R_{i}$.bsn. In Figure 7 (where $\left.R_{3}=R_{7}\right), R_{5} . b s n=3 ; R_{8} . b s n=3 ; R_{3} . b s n=7 ; R_{7} \cdot b s n=10 ; R_{2} . b s n=10$. If the regions in Figure 7 are the search regions of six top- $N$ queries, they can be considered as a cluster. It is sufficient to retrieve the tuples from $R_{10}$ using only one I/O request (one I/O request may incur multiple likely sequential I/Os), and then we can obtain the candidate sets from the BSRs for others. Note that $R_{3}=R_{7}, R_{3} \cdot b s n=7$, but $R_{7} \cdot b s n=10$. If $R_{3} \cdot b s n=7$ and $R_{7} \cdot b s n$ $=3$, there would be an infinite loop.

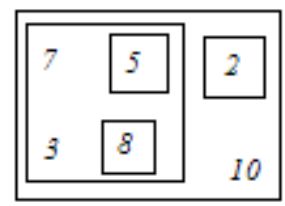

Fig.7. The best-super-region and best-super-region number

In addition, if we have $R_{l} \subset R_{2} \subset \ldots \subset R_{k}$, i.e., each $R_{i}$ is the best-super-region of $R_{i-l}$, then we can get the candidate sets for all regions from $R_{k}$. 


\subsubsection{Get Top- $N$ Tuples from Candidate Set (TTC)}

Algorithm TTC first computes the distance between a top- $N$ query $(Q, N)$ and every tuple in the candidate set of the query, and then identifies the $N$ tuples with the smallest distances. A heap structure can be used to keep track of the $N$ tuples with the smallest distances during the computation. Since this algorithm is straightforward, its detail will not be presented here.

If the candidate set has less than $N$ tuples, the search region of the query needs to be enlarged, which will be discussed in Section 4.4.2.

\subsection{Models of Clustering of Multiple Top- $N$ Queries}

In this section, we present three models of region clustering and discuss the clustering conditions for each model.

Let $\boldsymbol{T}=\left\{T_{i}: i=1, \ldots, m\right\}$ be a set of $m$ regions and $\boldsymbol{C}=\left\{H_{k}: k=0,1, \ldots, L\right\}$ be a subset of $\boldsymbol{T}$. If $\boldsymbol{C}$ satisfies some conditions, it is called a cluster. Suppose $S C R$ is the smallest containing region of $\mathcal{C}$.

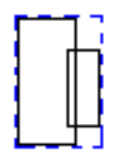

(a) I-clustering

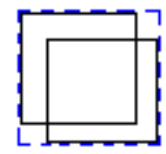

(b) C-clustering

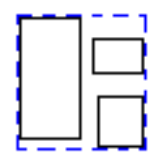

(c) S-clustering

Fig.8. Models of region clustering

\subsubsection{I-Clustering}

$\mathcal{C}$ is called an I-cluster (for Intersection based cluster) if it satisfies the following conditions:

(1) for each $0<j \leq L$, there is $0 \leq k<j$ such that $H_{k} \cap H_{j} \neq \phi$;

(2) $v\left(\cup_{k=0}^{L} H_{k}\right) / v\left(S C R\left(\left\{H_{k}\right\}\right)\right) \geq c_{1}$, where $c_{1}$ is a constant.

The first condition means that each region in $\mathcal{C}$ intersects with at least another region in $\mathcal{C}$. The second condition is to limit the lacuna of the $S C R$, making the regions in a cluster reasonably close to each other. Figure 8(a) shows an example of I-clustering.

The conditions of I-clustering are called I-cluster conditions, and $c_{1}$ is called I-cluster threshold. Since $0<$ $v\left(\cup_{k=0}^{L} H_{k}\right) / v\left(S C R\left(\left\{H_{k}\right\}\right)\right) \leq 1, c_{1} \in[0,1]$. It can be seen that in general a larger $c_{1}$ requires closer regions, which leads to more compact clusters (i.e., the lacuna of the $S C R$ is smaller). On the other hand, a larger $c_{1}$ reduces the applicability of I-clustering as fewer regions can satisfy the I-cluster conditions. In this paper, we determine an 
approximate optimal value of $c_{1}$ through training (see Section 5.2). Note that volume $v\left(\cup_{k=0}^{L} H_{k}\right)$ can be obtained using algorithm VUR.

\subsubsection{C-Clustering}

$\boldsymbol{C}$ is called a $\boldsymbol{C}$-cluster (for Center based cluster) if it satisfies the following conditions:

(1) for each $0<j \leq L$, there is $0 \leq k<j$ such that the center of $H_{j}$ is in $H_{k}$;

(2) $v\left(\cup_{k=0}^{L} H_{k}\right) / v\left(S C R\left(\left\{H_{k}\right\}\right)\right) \geq c_{2}$, where $c_{2}$ is a constant.

It is clear that $\mathrm{C}$-cluster is a special case of I-cluster and it requires the regions to have higher degree of overlap because of the condition that the center of one region is contained in another region. Figure 8(b) shows an example of C-clustering. The two conditions above are called $\boldsymbol{C}$-cluster conditions, and $c_{2}$ is called $\boldsymbol{C}$-cluster threshold. The impact of $c_{2}$ on $\mathrm{C}$-clustering is the same as that of $c_{1}$ on I-clustering. We also use training to determine an approximate optimal value for $c_{2}$.

\subsubsection{S-Clustering}

The set $\boldsymbol{C}=\left\{H_{k}: k=0,1, \ldots, L\right\}$ is called an $\boldsymbol{S}$-cluster (for Sum based cluster) if it satisfies the condition: $v\left(\operatorname{SCR}\left(\left\{H_{k}\right\}\right)\right) / \sum_{k=0}^{L} v\left(H_{k}\right)<c_{3}$, where $c_{3}$ is a constant.

The condition is called $\boldsymbol{S}$-cluster condition, and $\boldsymbol{c}_{3}$ is called $\boldsymbol{S}$-cluster threshold. Figure $8(\mathrm{c})$ shows an example of S-clustering and the three regions form an S-cluster. The value of $c_{3}$ reflects the degree of closeness of the regions. There is no clear upper bound for $c_{3}$. When $c_{3}<1$, according to the discussion about $\tau$ in Section 3 , some of these regions must overlap and they are already considered by either I-clustering or C-clustering. On the other hand, if $c_{3}$ $>3$, then these regions are likely to be too far apart to form effective clusters. Therefore, it is reasonable to consider $c_{3} \in[1,3]$. Training will again be used to determine an approximate optimal value for $c_{3}$.

\subsubsection{Comparison of the three clustering models}

Based on the above discussions about the three clustering models defined above, the relationships among the three models can be summarized as follows:

1. C-clustering tends to produce the most compact clusters (i.e., the lacuna of the $S C R$ tends to be the smallest), followed by I-clustering and then by S-clustering. 
2. S-clustering tends to be most applicable, followed by I-clustering and then by C-clustering.

\subsection{Cluster Search Regions}

Let $\mathbf{Q}=\left\{\left(Q_{l}, N_{l}\right), \ldots,\left(Q_{m}, N_{m}\right)\right\}$ be a set of $m$ top- $N$ queries and $R_{l}, \ldots, R_{m}$ be their respective search regions. In this section we discuss how to cluster the search regions in $\mathbf{R}=\left\{R_{k}: k=1, \ldots, m\right\}$ based on the basic algorithms and

clustering models described in Sections 4.1 and 4.2. For $R_{k}=\prod_{i=1}^{n}\left[a_{i}, b_{i}\right]$, we sometimes denote $R_{k} \cdot a_{i}=a_{i}$ and $R_{k} \cdot b_{i}=$ $b_{i}$. Also, we sometimes do not distinguish the top- $N$ query $\left(Q_{k}, N_{k}\right)$ and its search region $R_{k}$ in the following discussion. Suppose $\min \left(A_{i}\right)$ and $\max \left(A_{i}\right)$ are the minimum and maximum values of attribute $A_{i}$ of all tuples in a relation. Without loss of generality, for the set $\mathbf{R}$, if $R_{k} \cdot a_{i}<\min \left(A_{i}\right)$, let $R_{k} \cdot a_{i}:=\min \left(A_{i}\right)$, and if $R_{k} \cdot b_{i}>\max \left(A_{i}\right)$, let $R_{k} \cdot b_{i}:=\max \left(A_{i}\right)$.

Based on the relationships among the three clustering models (see Section 4.2.4), it seems natural to employ the following region clustering strategy. First, C-clustering is employed to the input search regions; next I-clustering is applied to those regions that are not clustered by C-clustering; and finally, S-clustering is used to cluster the remaining regions as much as possible. However, our experiments indicate that for some datasets, C-clustering is not effective because its relatively low applicability and after C-clustering is applied, it becomes more difficult to apply I-clustering to the remaining regions. Further analysis indicates that when the sizes of the search regions of the input top- $N$ queries are large, it is more likely that the regions can satisfy the center-in condition (i.e., the first condition) of the C-cluster conditions; as a result, C-clustering is more effective in such situations. On the other hand, when the search regions are small, C-clustering is not as effective as I-clustering.

Based on the above observation, we adopt the following general clustering strategy in this paper.

1. Apply either I-clustering or C-clustering to cluster the input regions based on the sizes of their search regions. Specifically, C-clustering is applied if the regions are large and I-clustering is applied if the regions are small.

2. Apply S-clustering to cluster the remaining regions.

For convenience, the I-clustering and C-clustering models will be called primary clustering models while the Sclustering model will be called a secondary clustering model. The size of a region can be estimated based on the distribution of data in a dataset, the query point and the value of $N$ in a top- $N$ query. 
More detailed description of our clustering algorithm is given below. Again $\mathbf{R}=\left\{R_{k}: k=1, \ldots, m\right\}$ is the set of search regions under consideration.

1. Label all the regions in $\mathbf{R}$ that have a super-region and remove them temporarily. For each $k$, if $R_{k}$ has a superregion, determine its best-super-region (BSR) (see Section 4.1.4) and save the BSR number, $R_{k} \cdot b s n$. Since we always place a region and its super regions in the same cluster, it is sufficient to consider how to cluster those that have no super regions.

Let $\boldsymbol{T}=\left\{T_{\mathrm{i}}\right\}$ denote the subset of the regions in $\mathbf{R}=\left\{R_{k}\right\}$ that have no super-regions. Moreover, let $m_{T}=|\boldsymbol{T}|$. Obviously, $m_{T} \leq|\mathbf{R}|$.

2. Obtain the Smallest Containing Region $(S C R)$ of all $\left\{T_{j}\right\}$. Denote $S C R \cdot a_{i}=\min _{1 \leq j \leq m_{T}}\left\{T_{j} \cdot a_{i}\right\}$ and $S C R \cdot b_{i}=$ $\max _{1 \leq j \leq m_{T}}\left\{T_{j} \cdot b_{i}\right\}$, where $i=1, \ldots, n$ and $n$ is the number of dimensions.

3. Partition the centers of the search regions in $\boldsymbol{T}$ into $K$ subsets, $1<K<m_{T}$, using Algorithm PPS (see Section 4.1.3). This effectively partitions the $S C R$ into $K$ sub-regions, $S_{1}, \ldots, S_{K}, 1<K<m_{T}$, and there are at most $m_{0}$ centers in each sub-region. The search regions in $\boldsymbol{T}$ whose centers are in the same sub-region are likely to be grouped into the same cluster.

4. Cluster the regions in $T$ using the clustering models described in Section 4.2. We first apply a primary clustering model (C-clustering or I-clustering) and then apply the secondary clustering model (S-clustering). We need a threshold $L_{0}$ as the maximum number of regions in a cluster $\mathcal{C}$. Because the VUR algorithm has a complexity of $\mathrm{O}\left(2^{m}\right)$, where $m$ is the number of regions, and we use VUR to calculate the volume of the unions of the search regions in $\mathcal{C}$ (see Section 4.2), $L_{0}$ should not be too large. We use $L_{0}=10$ in our experiments, which allows VUR to run fast.

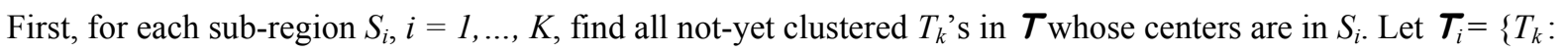
the center of $T_{k}$ is in $S_{i}, k=1, \ldots, L$, and $\left.l \leq L \leq m_{0}\right\}$ Second, arbitrarily select a region from $\boldsymbol{T}_{i}$, denote as $H_{0}$, and use it as the seed of a new cluster. Then find the next region $H_{1}$ from $\boldsymbol{T}-\left\{H_{0}\right\}$ that satisfies the clustering conditions of the clustering model being used. Repeat this process to find $H_{h}$ from $\boldsymbol{T}-\left\{H_{0}, H_{1}, \ldots, H_{h-1}\right\}$ until no remaining regions satisfy the clustering conditions of 
the clustering model or $h=L_{0}$ is reached. Thus we get a cluster $\boldsymbol{C}=\left\{H_{j}: j=0,1, \ldots, h-1, h \leq L_{0}\right\}$. If $\boldsymbol{T}_{i}$ is not empty, select another un-clustered region from $\boldsymbol{T}_{i}$ as the new seed and get another cluster by repeating the above process.

Let $\left\{\boldsymbol{C}_{i}, i=1, \ldots, M\right\}$ denote the clusters constructed above and build the smallest containing region $S C R_{i}=$ $\operatorname{SCR}\left(\boldsymbol{C}_{i}\right)$ for each cluster $\boldsymbol{C}_{i}, i=1, \ldots, M$. It is possible that some of the clusters may contain a single region, for example, when the selected seed cannot be clustered with the remaining regions.

5. Check if some of the un-clustered regions (i.e., clusters with a single region) can be added into an existing cluster. If there is such a region $T_{j} \subset S C R_{h}$, add $T_{j}$ into $\mathcal{C}_{h}$ (note that due to the constraint of the threshold $L_{0}$, it is possible that $T_{j}$ could not be added to a $\boldsymbol{C}_{i}$ in step 4). If there are multiple $S C R_{i}$ 's that contain such a $T_{j}$, place it in the cluster whose $S C R_{i}$ has the smallest volume. Therefore, it is possible that the size of the final cluster is more than $L_{0}$. The remaining un-clustered regions will be processed individually.

6. For each smallest containing region $S C R_{i}=S C R\left(\boldsymbol{C}_{i}\right)$, find its best-super-region (BSR) $S C R_{k}$ and save its BSR number $S C R_{i} \cdot b s n=k($ see Section 4.1.4).

Example 2. In Figure 9, $\left\{R_{i}: i=1, \ldots, 25\right\}$ is the set of search regions in 2-dimensinal space. Suppose these search regions are small. By applying I-clustering as the primary clustering model first, three clusters $\mathcal{C}_{1}=\left\{R_{1}, R_{2}\right\}$, $\mathcal{C}_{2}=\left\{R_{3}, R_{4}, R_{5}\right\}$ and $\mathcal{C}_{3}=\left\{R_{11}, R_{12}, R_{13}, R_{14}, R_{15}, R_{16}, R_{17}, R_{18}, R_{19}, R_{20}\right\}$ are formed. By applying S-clustering to the remaining regions, $\boldsymbol{\zeta}_{4}=\left\{R_{6}, R_{7}, R_{8}\right\}$ is formed. The smallest containing regions of $\boldsymbol{\zeta}_{1}, \boldsymbol{\zeta}_{2}, \boldsymbol{C}_{3}$ and $\boldsymbol{\zeta}_{4}$ are $S C R_{1}$, $S C R_{2}, S C R_{3}$ and $S C R_{4}$, respectively. $R_{9}$ is added into $\mathcal{C}_{1}$ since it is in the same cluster with its super-region $R_{1}$. By step 5, $R_{10}$ is added to $\zeta_{2}$ although it does not intersect with any of other regions in $\mathcal{C}_{2}$. Due to the constraint of $L_{0}$ ( $L_{0}=10$ ), $R_{24}$ was not added into $\zeta_{3}$ initially but added into $\zeta_{3}$ later because it is contained in $S C R_{3}$. Four regions $R_{21}$, $R_{22}, R_{23}$ and $R_{25}$ are single-region clusters; thus their corresponding queries will be evaluated independently. Although $S_{21} \cap S_{22} \neq \phi, S_{21}$ and $S_{22}$ do not form a cluster because they do not satisfy the second condition of Iclustering (see Section 4.2.2). Thus, for this example, the total number of $\mathrm{I} / \mathrm{O}$ requests to the database is 8 , one for each of $S C R_{1}, S C R_{2}, S C R_{3}, S C R_{4}, R_{21}, R_{22}, R_{23}$ and $R_{25}$. 


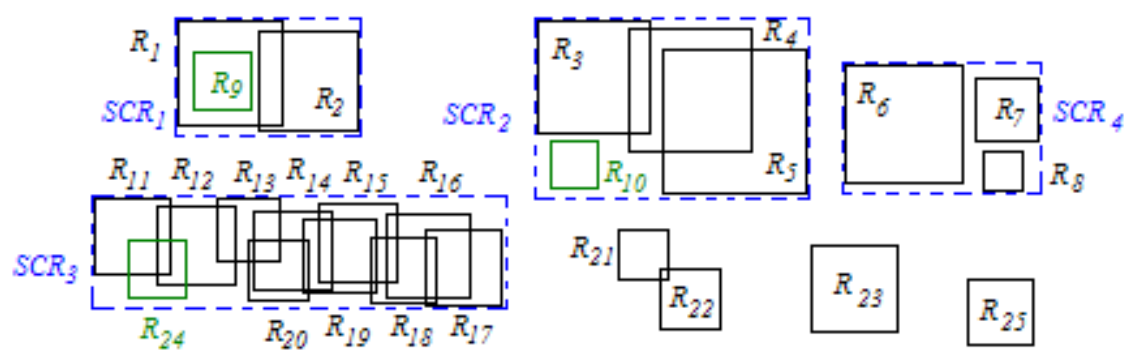

Fig.9. An example of clusters for $\left\{R_{i}: i=1, \ldots, 25\right\}$

\subsection{Top- $N$ Tuple Retrieval}

\subsubsection{Search top- $N$ tuples}

For obtaining the sets of top- $N$ tuples for $m$ queries, we consider two cases, one is for the clustered queries, and the other is for individual queries.

Case 1: clustered queries. There are three steps.

Step 1. From $\left\{S C R_{i}\right\}$, find the first $S C R$ that has no super region, denote it as $S C R_{i_{0}}=\prod_{i=1}^{n}\left[a_{i}, b_{i}\right]$. Retrieve all tuples $\left\{t^{(i)}: i=1, \ldots, M\right\}$ from it using a simple selection range query:

$$
\operatorname{SELECT} * \operatorname{FROM} R \text { WHERE }\left(a_{1} \leq A_{l} \leq b_{1}\right) \text { AND } \ldots \text { AND }\left(a_{n} \leq A_{n} \leq b_{n}\right)
$$

For each search region $R$ in $\boldsymbol{C}_{i_{0}}$ (therefore $R \subset S C R_{i_{0}}$ ),

(a) if $R$ has no super region, search the candidate set of $R$ from $\left\{t^{(i)}: i=1, \ldots, M\right\}$, and get the top- $N$ tuples using algorithm TTC.

(b) else (i.e. $R$ has a super region), find out its best-super-region BSR, search the candidate set from the BSR and get the top- $N$ tuples by using algorithm TTC. Note that getting the best-super-region may be a recursive process if BSR has its own super region.

Step 2. For each $S C R_{i}, i>i_{0}$, that has no super region,

(a) if there is no $S C R_{j}\left(i_{0} \leq j<i\right)$ that intersects with $S C R_{i}$, follow the same procedure as Step 1.

(b) else, among all $S C R_{j}$ 's $\left(i_{0} \leq j<i\right)$ that intersect with $S C R_{i}$, find the one, denoted $S C R_{j_{0}}$, that has the largest intersection. Then calculate the difference of the two regions using algorithm DTR: $S C R_{i}-S C R_{j_{0}}$ and obtain all tuples $\left\{t^{(i)}: i=1, \ldots, M\right\}$ in $S C R_{i}$ using algorithm CST. Get the top- $N$ tuples as Step 1 using TTC.

Step 3. For each $S C R_{i}, i \geq 1$, that has a super region, find its best-super-region $S C R_{k}$, and get all the tuples in $S C R_{i}$ from $S C R_{k}$. Then follow the same procedures as Step 1(a) and Step 1(b) for each region $S C R_{i}$. 


\section{Case 2: individual queries.}

Step 1. For each $R_{i}$, if it has no super region, find all the clustered $R_{j}$ 's that intersect with $R_{i}$, and let $R_{j_{0}}$ be the one with the largest intersection. All candidates in $R_{j_{0}}$ have been retrieved in Case 1 above. Calculate $R_{i}-R_{j_{0}}$ using algorithm DTR, and obtain candidates of $R_{i}$ using CST, then get the top- $N$ tuples using TTC.

Step 2. If $R_{i}$ has a super region, it is sufficient to get all the candidates in $R_{i}$ from its best-super-region $R_{k}$ and then obtain the top- $N$ tuples for $R_{i}$ using TTC.

There are two special situations in Case 1 and Case 2. If two queries are the same, it suffices to obtain the top- $N$ tuples only one time; if a search region is the same as its best-super-region, it is sufficient to get the candidates once.

\subsubsection{Guarantee exact top- $N$ tuples}

If there are not at least $N$ tuples in the search region $R$ of a top- $N$ query $(Q, N)$, i.e. $|R|<N$, then the process of Section 4.4.1 cannot guarantee to get the top- $N$ tuples. Two cases are considered as follows.

1. $R$ has a best-super-region $R_{k}$ or $S C R$, and the size of $R_{k}$ or $S C R$ contains at least $N$ tuples. Suppose $|R|=N^{\prime}$, take $N$ - $N^{\prime}$ tuples from $R_{k}-R$ or $S C R-R$, and calculate the distances between those tuples and the query $Q$. Now we use the maximum distance to define the new search region for $Q$ and this search region guarantees at least $N$ tuples will be retrieved. Get the top- $N$ tuples-using algorithm TTC.

2. $R$ has no super region or the number of tuples in its super region is also less than $N$. Use an existing method such as the learning-based method in [43] or the histogram-based method in [5], to determine a larger search region that contains at least $N$ tuples.

\section{EXPERIMENTAL RESULTS}

In this section, we report our experimental results and compare our Region Clustering Method (RCM) against the Naïve Method (NM) for evaluating multiple top- $N$ queries. Our experiments are carried out using Microsoft's SQL Server 2000 and $\mathrm{VC}++6.0$ on a PC with Windows XP and a Pentium 4 processor with $2.8 \mathrm{GHz} \mathrm{CPU}$ and $768 \mathrm{MB}$ memory.

\subsection{Data Sets and Preparations}

The datasets we used include data of both low dimensionality (2, 3, and 4 dimensions) and high dimensionality (25, 50, and 104 dimensions). For low-dimensional datasets, both synthetic and real datasets used in [5] are used. The real datasets include Census2D and Census3D (both with 210,138 tuples), and Cover4D (581,010 tuples). The 
synthetic datasets are Gauss3D (500,000 tuples) and Array3D (507,701 tuples). In the names of all datasets, suffix " $\mathrm{nD}$ " indicates that the dataset has $n$ dimensions. For high-dimensional datasets, real datasets derived from LSI are used in our experiments. They have 20,000 tuples and the same 25, 50 and 104 attributes as used in [11] are used to create datasets of 25, 50 and 104 dimensions, denoted by Lsi25D, Lsi50D and Lsi104D, respectively.

Each dataset uses three training-workloads $t w_{1}, t w_{2}$ and $t w_{3}$, and three test workloads $w_{1}, w_{2}$ and $w_{3}$. Each trainingworkload includes 100 queries and each test workload includes 2,000 queries that are the tuples randomly selected from the dataset used respectively. Three training-workloads will be used to determine the clustering models and their threshold values, and three test workloads will be used to report our experimental results, which are the average of the results of the three test workloads.

Two top- $N$ query evaluation techniques are used to construct the search region of a top- $N$ query:

1. Optimum (Opt) technique [5]: As a baseline, we consider the ideal technique that uses the smallest search region containing the actual top- $N$ tuples for a given query. The smallest search region is obtained using the sequential scan technique. That is, first obtain the top- $N$ tuples from scanning the entire dataset, and then construct the search region that just contains the top- $N$ tuples for each query.

2. Learning-based $(L B)$ technique [43]: For a given dataset $\mathrm{D}$, a set of data points is randomly selected from $\mathrm{D}$ and these data points are used as sample query points. For each such query point, a top-N query $\left(Q_{i}, N_{i}\right)$ is generated and a profile is created for it. The profile contains the search distance $r_{i}$ of the smallest search region of $\left(Q_{i}, N_{i}\right)$ (obtained using the sequential scan technique) and the number of tuples in this search region. Once the profiles are created and saved, the learning-based technique uses them to estimate the search engine for any new top- $N$ query, $(Q, N)$. More specifically, we first identify the profiles whose query points are closest to $Q$, then we use the information in these profiles to estimate the local density of $Q$, and finally we construct the search region for $(Q, N)$ (please refer to [43] for more details about this method). The experimental results reported in [43] indicate that using less than 1,000 sample queries to create the profiles is sufficient. For the experiments conducted in this paper, 178, 218, 250, 833, 909 and 954 profiles are created for datasets of 2, 3, 4, 25, 50 and 104 dimensions, respectively. These numbers are determined based on the consideration that the total size of the profiles does not exceed the size of the histogram used in [5] and the size of sampling set used in [11] when these methods are compared using the same dataset [43]. 
For any given top-N query, the search region generated by the Opt method is usually smaller than that generated by the LB method because the former is the smallest possible. With smaller search regions, the likelihood of overlap among them will be reduced. Consequently, I-Clustering and C-Clustering may become less effective.

In our experiments, we report results based on a default setting. This default setting uses a 2,000-query workload. For low dimensional datasets (2,3, and 4 dimensions), the default setting has $N=100$ (i.e., retrieve top 100 tuples for each query) and the distance function is the maximum distance (i.e., $\mathrm{L}_{\infty}$-norm distance); for high dimensional datasets (25, 50, and 104 dimensions), the default setting has $N=20$ and the distance function is Euclidean distance (i.e., $\mathrm{L}_{2}$-norm distance) [43].

The following measures are used in our experiments:

- The elapsed time (millisecond, ms) used to obtain top-N tuples: The sum of the times needed to retrieve the top$N$ tuples from the respective dataset for all queries. The time for constructing the search regions is not included because this time is the same for both Naïve and RCM query evaluation strategies. In addition, the time to construct the search regions is relatively small compared to the time needed to retrieve the tuples from the regions. For example, it takes about $3 \mathrm{~ms}$ to construct one search region and about $150 \mathrm{~ms}$ to retrieve the data from a region for Lsi25D.

- $\quad$ The number of I/Os: It is the total number of $\mathrm{I} / \mathrm{O}$ operations for accessing the database.

- $\quad$ The extra time used by RCM (millisecond, $m s$ ): It is the sum of the times needed to perform the clustering using our algorithms, i.e., it corresponds to the $\delta$ in Formula (2) in Section 3.

- The number of tuples retrieved: The total number of tuples retrieved in order to obtain the top- $N$ tuples from the respective dataset for all queries. A smaller number of tuples retrieved indicates a better efficiency.

The values of the above four measures depend on the following set of factors $\{m, \mathrm{D}, \mathrm{T}, \mathrm{A}, \mathrm{W}\}$, where $m$ : The number of top- $N$ queries to be evaluated concurrently. The following values are considered: $1,4,10,40$, $100,400,1000$ and 2000.

D: The dataset used. Its valid "values" include Census2D, Census3D, Array3D, Gauss3D, Cover4D, Lsi25D, Lsi50D, and Lsi104D.

T: The technique used to obtain the search region of a top- $N$ query. Its valid "values" are LB (Learning-based technique) and Opt (Optimum technique). 
A: The algorithm used to retrieve the top- $N$ tuples for the $m$ top- $N$ queries. It's either RCM or NM. The naïve method (NM) is frequently used as the baseline to evaluate other methods of processing multiple queries [16] because it is simple, easy to understand, and generally usable.

$\mathrm{W}$ : The workloads: three workloads, $w_{1}, w_{2}$ and $w_{3}$, are used to get the original values of the performance measures, and then their average is taken to obtain the reported results.

Because the elapsed time and the extra time do not include the time needed to construct the search regions (the $\mathrm{RC}($ ) in Formulas (1) and (2) in Section 3), from Formulas (1) and (2) in Section 3, we have the following formulas:

Elapsed-time $(m, \mathrm{D}, \mathrm{T}, \mathbf{N M}, \mathrm{W})=\left(\operatorname{IO-time}\left(Q_{1}, N_{l}\right)+S\right.$-time $\left.\left(Q_{1}, N_{l}\right)\right)+\ldots$

$$
+\left(\text { IO-time }\left(Q_{m}, N_{m}\right)+S \text {-time }\left(Q_{m}, N_{m}\right)\right)
$$

Elapsed-time $(m, \mathrm{D}, \mathrm{T}, \mathbf{R C M}, \mathrm{W})=\left(\right.$ IO-time $\left(S C R_{I}\right)+\ldots+I O$-time $\left.\left(S C R_{M}\right)\right)+$

$$
\left(S \text {-time }\left(Q_{l}, N_{l}\right)+\ldots+\text { S-time }\left(Q_{m}, N_{m}\right)\right)+\operatorname{Extra-time}(m, \mathrm{D}, \mathrm{T}, \mathbf{R C M}, \mathrm{W})
$$

where IO-time ( ) is the elapsed time of the I/O operations for accessing the database and S-time( ) is the elapsed time of sorting the results. In (2'), $\left\{S C R_{k}: k=1, . ., M\right\}$ includes all single-region clusters.

It is well known that the cost of all the algorithms that we consider depend on the size of the buffer in main memory. When presenting cost estimates we generally assume the worst-case scenario [34] and let the size of the buffer be as small as possible. In Microsoft's SQL Server 2000, we configure the 'max server memory' to be 5MB for Lsi50D, 8MB for Lsi104D, and 4MB for the other datasets. Note that 4MB is the smallest value for the 'max server memory' in Microsoft's SQL Server 2000.

\subsection{Determining Clustering Model and Thresholds by Training}

Each of the three clustering models we introduced in Section 4.2 has a threshold whose value can affect the quality of the produced clusters. From the discussion in Section 4.2, we have $0 \leq c_{1} \leq 1,0 \leq c_{2} \leq 1$, and $1 \leq c_{3} \leq 3$, where $c_{1}, c_{2}$ and $c_{3}$ are the thresholds for I-clustering, C-clustering and S-clustering, respectively. In this section, we discuss how to obtain the appropriate values for these thresholds for each dataset.

Our idea is to use the three training workloads associated with each dataset to perform this task. Let $\mathrm{D}$ be a dataset under consideration and $t w_{1}, t w_{2}$ and $t w_{3}$ be the associated training workloads. Our training process is as follows. First, we obtain two sets of search regions for the queries in each training workload, one based on the LB technique and the other based on the Opt technique. Next, for each set of search regions, we run two versions of our RCM 
algorithm, one employs only the I-clustering and the other uses only the C-clustering (S-clustering will not be used in both cases), with different values of $c_{1}$ and $c_{2}$, respectively. Based on the performances of these experiments, we find the best values for $c_{1}$ and $c_{2}$ and determine which of the two primary clustering models (i.e., I-clustering and Cclustering) is better based on their best threshold values. Finally, we add the S-clustering to the RCM algorithm for each best case and try different values of $c_{3}$ between 1 and 3 to determine the best value for $c_{3}$.

Example 3. Consider the dataset Gauss3D in Section 5.1. For each training-workload $t w_{i}, i=1,2,3$, use each of the LB and Opt techniques to get 100 search regions, respectively. Then apply I-clustering and C-clustering in the RCM algorithm with $c_{1}$ and $c_{2}$ being $0,0.05,0.1,0.15, \ldots, 0.95$, and 1 , respectively. Based on the number of I/Os we obtained for each run using the I-clustering, we compute the average of the number of I/O requests for the 3 training workloads and then the ratios of the number of I/Os of RCM to NM:

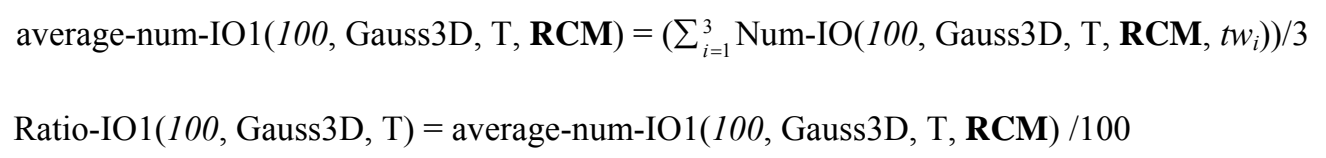

Similarly, based on the number of tuples retrieved we obtained for each run using the I-clustering, we compute the average of the number of tuples retrieved for the 3 training workloads and then the ratios of the number of tuples retrieved by RCM to that by NM:

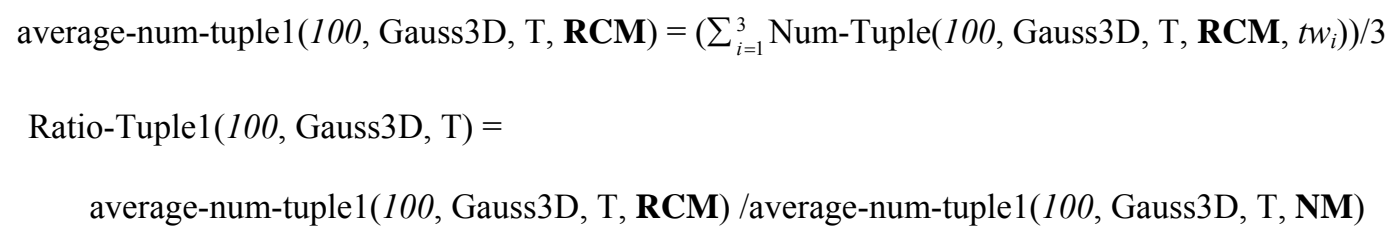

We can repeat the above computations for C-clustering. Let "average-num-IO2", "Ratio-IO2", "average-numtuple2" and "Ratio-Tuple2" denote the corresponding averages and ratios for C-clustering.

Figure 10(a) shows the results for different values of $c_{1}$ and $c_{2}$ using the regions constructed by the LB technique. Obviously, 0.5 is a pivotal point with I-clustering; when $c_{1}$ and $c_{2}$ are 0.5 , the number of $I / O s$ of I-clustering is less than that of C-clustering, and the numbers of tuples retrieved are almost the same for both clustering models. 


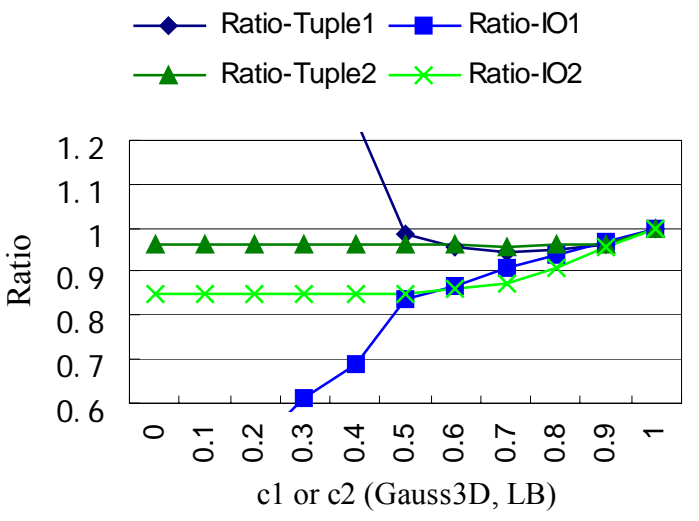

(a) The regions constructed by LB technique
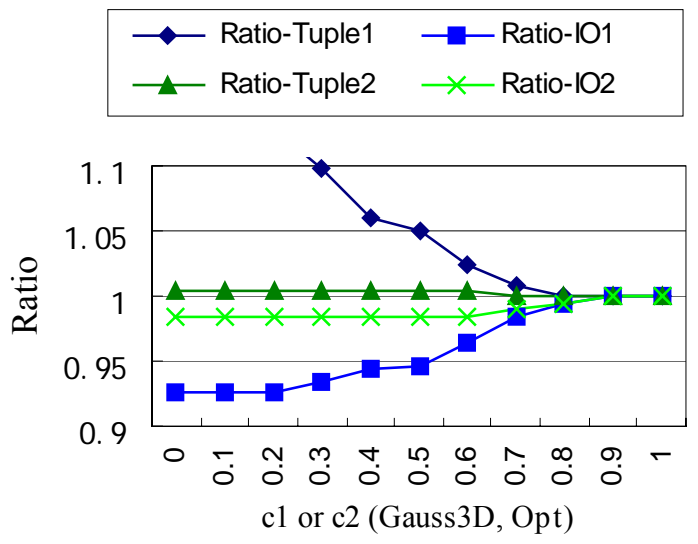

(b) The regions constructed by Opt technique

Fig.10. Ratios of the number of $\mathrm{I} / \mathrm{O}$ and ratios of the number of tuples retrieved by training workloads for Gauss3D

Figure 10(b) shows the results where the search regions are constructed using the Opt technique. We can see that when $c_{1}$ and $c_{2}$ are 0.5 , the ratio of tuples retrieved using I-clustering is about 1.05 , and the radio of I/Os of Iclustering is less than that of C-clustering. Since the number of I/Os plays a bigger role in efficiency, we choose Iclustering with threshold $c_{1}=0.5$ as the primary clustering model for dataset Gauss $3 \mathrm{D}$.

Table 1 lists the primary clustering model and the approximate optimal thresholds that are obtained for each dataset using the training workloads $t w_{1}, t w_{2}$, and $t w_{3}$. All experimental results to be reported in the subsequent sections are based on the thresholds in Table 1.

Table 1: Primary clustering model and thresholds for all datasets

\begin{tabular}{|c|c|c|c|}
\hline \multirow{2}{*}{ Dataset } & \multicolumn{2}{|c|}{ Primary Model } & \multirow{2}{*}{$\begin{array}{l}\text { Secondary Model } \\
\text { ( S-clustering ) }\end{array}$} \\
\hline & I-clustering & C-clustering & \\
\hline Census2D & $c_{1}=0.25$ & - & $c_{3}=1.5$ \\
\hline Census3D & $c_{1}=0.25$ & - & $c_{3}=1.5$ \\
\hline Gauss3D & $c_{1}=0.5$ & - & $c_{3}=2$ \\
\hline Array3D & - & $c_{2}=0.5$ & $c_{3}=2$ \\
\hline Cover4D & $c_{1}=0.25$ & - & $c_{3}=1.5$ \\
\hline Lsi25D & - & $c_{2}=0.25$ & $c_{3}=1$ \\
\hline Lsi50D & - & $c_{2}=0.1$ & $c_{3}=1$ \\
\hline Lsi104D & - & $c_{2}=0.0$ & $c_{3}=1$ \\
\hline
\end{tabular}

Table 1: Primary clustering model and thresholds for all datasets 


\subsection{Performance Comparison}

Using the thresholds in Table 1, for each of the 8 datasets $\mathrm{D}$, each of the three test workloads, and one of the two techniques LB and Opt for generating search regions, we first obtain 2,000 search regions corresponding to the 2,000 queries, then we use $m$ regions(s) to carry out one group of experiments, where $m$ takes one of the following 8 values: $1,4,10,40,100,400,1000$ and 2000 . Thus, there are $8 * 3 * 2 * 8=384$ groups of experimental results.

\subsubsection{Comparison of the Elapsed Time}

We use the ratio of RCM's performance to NM's performance to compare RCM and NM. Denote

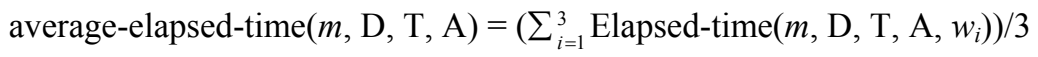

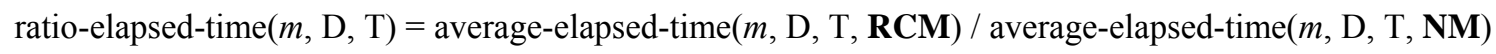

Figure 11(a) shows that, using the regions constructed by the LB-technique, ratio-elapsed-time $<1.0$ for all datasets when $m \geq 10$, except for Census3D which has the ratio $=1.0$ when $m=10$, and ratio-elapsed-time $\leq 0.82$ for all datasets when $m=100$, i.e., RCM is more efficient than $\mathbf{N M}$ when $m \geq 10$. Even when $m<10$, we have Ratiotime $\approx 1.0$. This means that $\mathbf{R C M}$ is generally usable for all $m$.

When $m=100$, Figure 11(a) shows that there are four datasets (Cover4D, Lsi25D, Lsi50D, and Lsi104D) with ratio-elapsed-time $<0.62$ (for Lsi25D and Lsi50D, the ratios are 0.33 and 0.28 respectively), that is, the elapsed time of RCM is less than $62 \%$ of that of NM for these datasets. Therefore, our RCM can save the time by at least $38 \%$ for these four datasets, and save the time by $67 \%$ and $72 \%$ for datasets Lsi25D and Lsi50D, respectively. The ratios for the other four datasets (Census2D, Census3D, Array3D and Gauss3D) are between 0.62 and 0.82 .

We can see that more savings on elapsed time can be achieved with the increase of the number of queries from 400 to 2000 . When $m=2000$, in the worst case, RCM can save the time by $50 \%$ for Gauss $3 \mathrm{D}$; but in the best case, RCM can save the time by nearly $90 \%$ for Census2D, Census3D and Lsi25D.

We should point out that when the number of queries $m$ becomes sufficiently large, the I/O cost for evaluating most additional queries would become zero. This occurs when the SCRs of the formed clusters cover almost the entire space, and as a result, the search region of any additional query considered will likely be contained in one of the existing SCRs, leading to no additional I/O operation. In other words, when $m$ becomes sufficiently large, the total I/O cost approaches a constant when RCM is used. For example, for Lsi25D, the number of I/O operations by RCM is 70 for both $m=1000$ and $m=2000$. Moreover, if we assume that every query incurs the same I/O cost, IO- 
time, and the same sorting cost, $S$-time, based on the naïve method, then the ratio-elapsed-time of RCM to NM is bounded by $S$-time/(IO-time $+S$-time $)$, which represents the extreme case when RCM incurs no I/O cost.

Figure 11(b) shows the results when the search regions are constructed using the Opt technique. When $m=100$, all ratios are between 0.52 and 0.9 , except for cover4D which has the ratio $=0.95$. Note that the Opt technique is the theoretical best for evaluating individual top- $N$ queries (i.e., using $\mathbf{N M}$ ) and it cannot be implemented in practice. The results in Figure 11(b) show that RCM can outperform NM even when the smallest search region is used for each query; for $m=2000$, all ratios are between 0.3 and 0.7 .

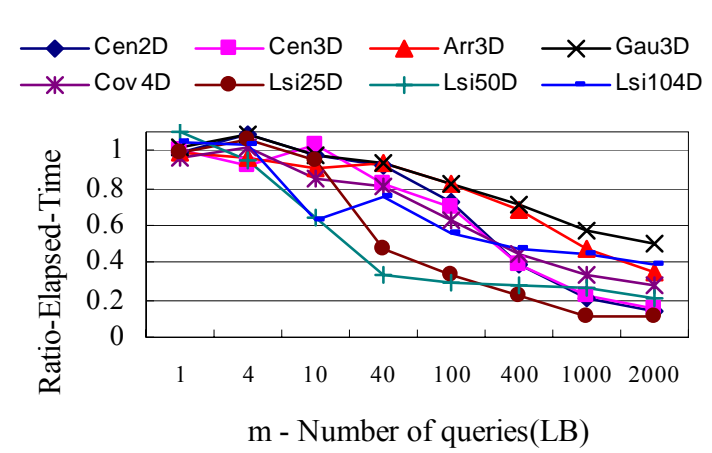

(a) The regions constructed by LB technique

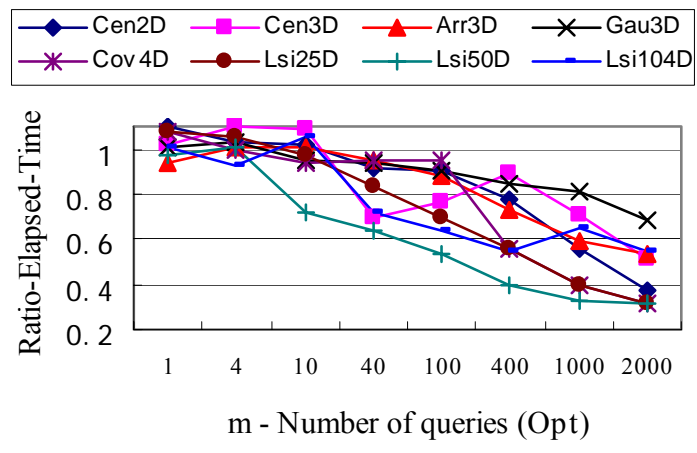

(b) The regions constructed by Opt technique

Fig.11. The ratios of elapsed times of RCM to NM

In Figures 11(a) and (b), the ratios should be close to 1.0 if no cluster contains two or more regions. However, there are some ratios that are greater than 1.0, say, when $m=1$ for Lsi50D in Figure 11(a) and when $m=4$ for Census3D in Figure 11(b). The primary reason is that the total elapsed time is very small in these cases so that a small random perturbation can cause the ratio to have a large change. The effect of random perturbation diminishes when $m$ becomes larger. This will also be discussed in Section 5.3.3.

\subsubsection{Comparison of the Number of I/Os}

The total number of $\mathrm{I} / \mathrm{O}$ requests for accessing the database plays an important role in the total response time of queries. In fact, $\mathrm{I} / \mathrm{O}$ cost of accessing the database is usually the dominating cost of processing database queries. We denote

average-num-IO $(m, \mathrm{D}, \mathrm{T}, \mathrm{A})=\left(\sum_{i=1}^{3} \operatorname{Num}-\mathrm{IO}\left(m, \mathrm{D}, \mathrm{T}, \mathrm{A}, w_{i}\right)\right) / 3$.

Because average-num- $\mathrm{IO}(m, \mathrm{D}, \mathrm{T}, \mathbf{N M})$ is always $m$, we have

ratio-num- $\mathrm{IO}(m, \mathrm{D}, \mathrm{T})=$ average-num- $\mathrm{IO}(m, \mathrm{D}, \mathrm{T}, \mathbf{R C M}) / \operatorname{average-num}-\mathrm{IO}(m, \mathrm{D}, \mathrm{T}, \mathbf{N M})$ 


$$
=\text { average-num- } \mathrm{IO}(m, \mathrm{D}, \mathrm{T}, \mathbf{R C M}) / m \text {. }
$$

Figure 12(a) shows that, using the search regions constructed by the LB technique, ratio-num-IO $<1.0$ for all datasets when $m \geq 10$, except for Census3D which has ratio 1.0 when $m=10$. For $m=100$, the ratio is less than 0.6 for four datasets (Cover4D, Lsi25D, Lsi50D and Lsi104D) and between 0.6 and 0.8 for the other four datasets. As mentioned in Section 5.3.1, when the number of queries becomes sufficiently large, the total I/O cost of RCM will approach a constant. Consequently, the ratio-num-IO will approach zero when $m$ becomes larger and larger because the total I/O cost of NM will keep on increasing as $m$ increases.

Figure 12(b) shows the case when the search regions are constructed using the Opt technique. When $m \geq 40$, the ratios are less than $<1.0$ for all datasets. Because the Opt technique yields the smallest search regions, which means there is less chance to form clusters. As a result, the ratio-num-IO has a slower speed of approaching zero compared with the case when the LB technique is used to generate search regions.

Comparing Figures 11(a)-(b) with Figures 12(a)-(b), respectively, we can see that the curves of ratio-elapsed-time and the curves of ratio-num-IO have similar trends. This indicates that the number of I/O requests has a direct and proportional impact on the elapsed time for sufficiently large $m$.

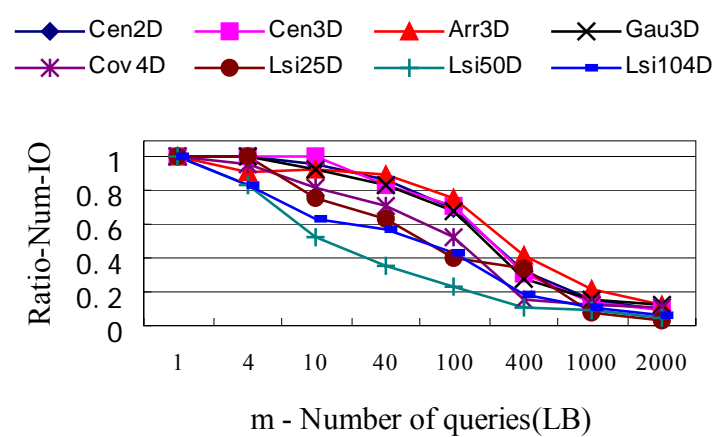

(a) The regions constructed by LB technique
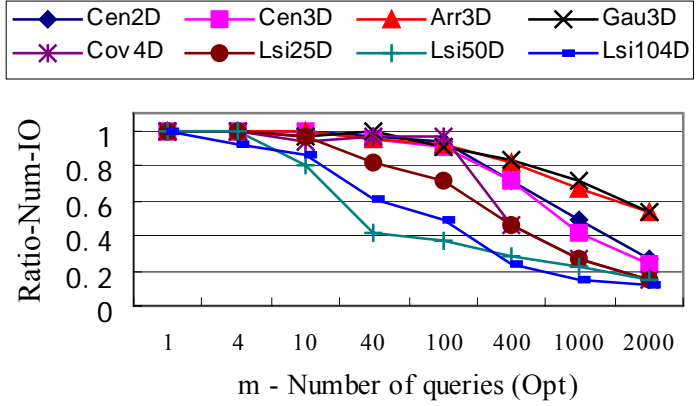

(b) The regions constructed by Opt technique

Fig.12. Ratios of the number of I/Os of RCM to NM

\subsubsection{Comparison Between the Extra Elapsed Time and Total Elapsed Time Using RCM}

In this subsection, we discuss the extra elapsed time for RCM. From Formula (2') in Section 5.1 and the first formula in Section 5.3.1, we have:

$\operatorname{extra-time}(m, \mathrm{D}, \mathrm{T}, \mathbf{R C M}, \mathrm{W})=\operatorname{elapsed-time}(m, \mathrm{D}, \mathrm{T}, \mathbf{R C M}, \mathrm{W})-$

$\left(\left(\operatorname{IO}\right.\right.$-time $\left(S C R_{l}\right)+\ldots+\operatorname{IO}$-time $\left.\left(S C R_{k}\right)\right)+\left(\operatorname{S-time}\left(Q_{l}, N_{l}\right)+\ldots+S\right.$-time $\left.\left.\left(Q_{m}, N_{m}\right)\right)\right)$ 
average-elapsed-time $(m, \mathrm{D}, \mathrm{T}, \mathbf{R C M})=\left(\sum_{i=1}^{3} \operatorname{elapsed-time}\left(m, \mathrm{D}, \mathrm{T}, \mathbf{R C M}, w_{i}\right)\right) / 3$

We denote

average-extra-time $(m, \mathrm{D}, \mathrm{T}, \mathbf{R C M})=\left(\sum_{i=1}^{3} \operatorname{extra-time}\left(m, \mathrm{D}, \mathrm{T}, \mathbf{R C M}, w_{i}\right)\right) / 3$

$\operatorname{ratio-extra-time}(m, \mathrm{D}, \mathrm{T})=\operatorname{average-extra-time}(m, \mathrm{D}, \mathrm{T}, \mathbf{R C M}) / \operatorname{average-elapsed-time}(m, \mathrm{D}, \mathrm{T}, \mathbf{R C M})$

From Figures 13(a) and (b), we can see that all the values for ratio-extra-time are very small. In fact, none of the ratios exceed 0.04 and 0.05 , respectively.

We can see that the curve of ratio-extra-time rises with the increase of the number of queries when $m \geq 100$ for each dataset because of two factors, i.e., the extra time will become larger and the total elapsed time of RCM increases more slowly as the total I/O cost approaches a constant. Nevertheless, the extra time remains very small compared with the total elapsed time of RCM. When $m$ is small $(m \leq 10)$, the total elapsed time is very small; therefore, a small random perturbation can lead to the curve of ratio-extra-time to have a large change.

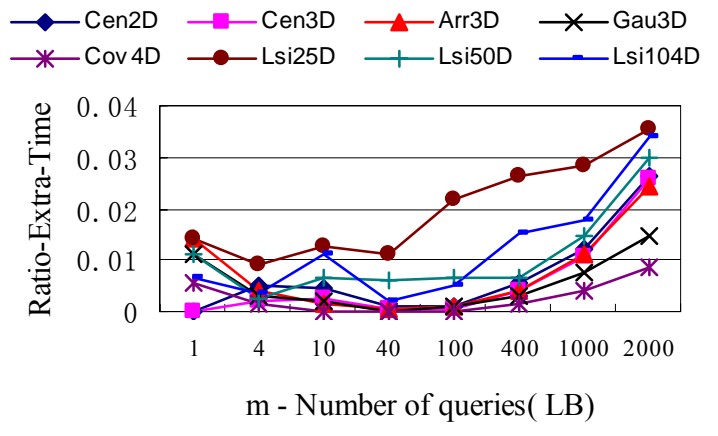

(a) The regions constructed by LB technique

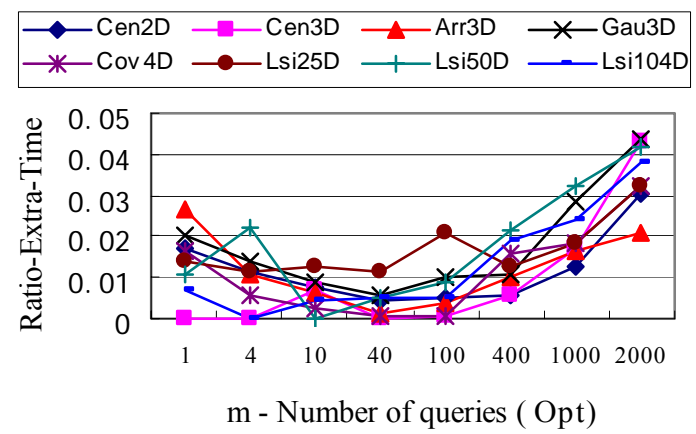

(b) The regions constructed by Opt technique

\section{Fig.13. The ratios of extra time to total elapsed time}

\subsubsection{Comparison of the Number of Tuples Retrieved}

With the requirement that the top- $N$ tuples for each query must be retrieved, an evaluation method is usually more efficient if it retrieves a smaller number of tuples because this means less useless tuples would be retrieved. We denote

average-num-tuple $(m, \mathrm{D}, \mathrm{T}, \mathrm{A})=\left(\sum_{i=1}^{3} \operatorname{Number}-\operatorname{Tuple}\left(m, \mathrm{D}, \mathrm{T}, \mathrm{A}, w_{i}\right)\right) / 3$

ratio-num-tuple $(\mathrm{m}, \mathrm{D}, \mathrm{T})$ = average-num-tuple $(\mathrm{m}, \mathrm{D}, \mathrm{T}, \mathbf{R C M})$ / average-num-tuple (m, D, T, NM) 

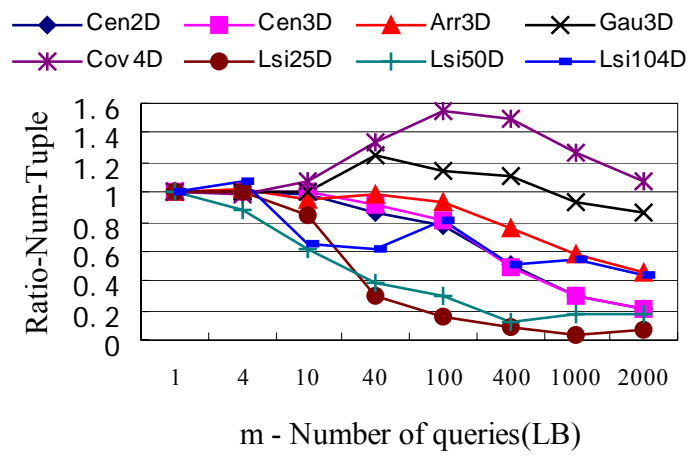

(a) The regions constructed by LB technique

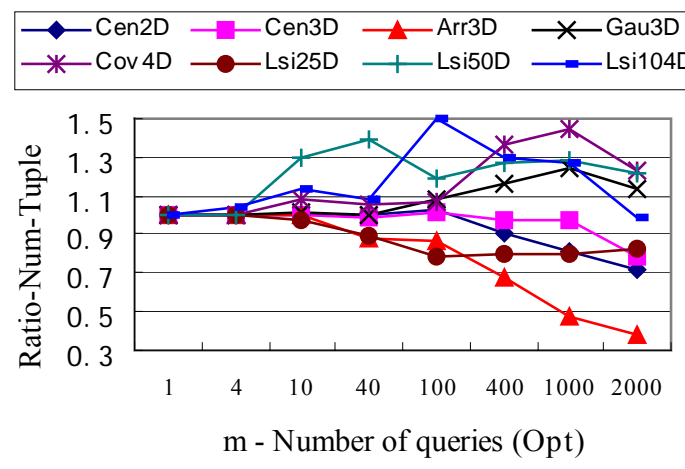

(b) The regions constructed by Opt technique

Fig.14. The ratios of the number of all tuples retrieved by RCM to that by NM

In Figure 14(a), for regions generated by the LB technique, the curves of ratio-num-tuple are either near 1 or below 1 except for datasets Cover4D and Gauss3D. Actually for most datasets, when $m \geq 40$, the ratios are less than 1. In other words, other than for datasets Cover4D and Gauss3D, the number of tuples retrieved by the RCM method is either the same as (usually for small $m$ ) or less than (usually for larger $m$ ) that by the Naïve Method. The reason for the exception of Cover4D and Gauss3D is due to the nature of the two datasets (their data distributions) and their parameters (see Table 1 in Section 5.2). The two datasets all use I-clustering as their primary clustering model, which tends to yield larger lacunas in the SCRs (smallest containing regions) (see Section 4.2), causing more tuples to be not in the search regions of the queries.

In Figure 14(b), for regions generated by the Opt technique, when $m>10$, the curves of ratio-num-tuple are either near 1 or below 1 except for datasets Cover4D, Gauss3D, Lsi50D and Lsi104D. So the number of all tuples retrieved by the RCM method is almost equal to that by the Naïve Method even when optimal regions are used except for datasets Cover4D, Gauss3D, Lsi50D and Lsi104D. The reasons for the exceptions are that smaller clustering thresholds tend to generate larger lacunas in the $S C R s$, and $c_{2}$ is 0.1 and 0.0 for Lsi50D and Lsi104D, respectively (see Section 4.2 and Table 1 in Section 5.2). Note that the number of all tuples retrieved by the Naïve Method is the smallest possible for regions generated by the Opt technique. This shows that RCM performs very well.

\section{CONCLUSIONS}

In this paper, we proposed a new method for evaluating multiple top- $N$ queries concurrently over a relational database. The basic idea of this method is region clustering that groups the search regions of individual top- $N$ 
queries into larger regions and retrieves the tuples from the larger regions. This method tends to avoid accessing the same region multiple times and reduce the number of random I/O accesses to the underlying databases. Our region clustering method is based on three basic clustering models, namely I-clustering, C-clustering and S-clustering, with different properties in terms of the compactness of the generated clusters and the applicability to region clustering. We carried out extensive experiments to evaluate the effectiveness of the proposed method based on eight different datasets with dimensionality ranging from 2 to 104, the number of concurrent queries considered ranging from 1 to 2,000, and the initial search regions obtained using two techniques (Learning-based and optimum). Our experimental results showed that our region clustering method yielded significant cost savings over the naïve onequery-at-a-time method for both low-dimensional and high-dimensional data. When 2,000 concurrent queries are considered and when more realistic initial search regions (the ones generated by the learning-based method) are used, the newly proposed method reduced the elapsed-time of the naïve method by 50 to $90 \%$ with most saving coming from the saving on $\mathrm{I} / \mathrm{O}$ cost.

We believe our method is directly applicable to evaluating multiple range queries in multi-dimensional spaces. Since unlike top- $N$ queries, traditional range queries do not involve sorting the final results, the region clustering method may have more relative advantage in evaluating multiple range queries than evaluating multiple top- $N$ queries. In the future, we plan to apply this method to process multiple traditional range queries and evaluate its effectiveness.

The experiments conducted in this paper assumed that a single server evaluates all the queries. As such, the results are directly applicable to a client/server environment where the server evaluates all the queries sent in from different clients. It would be interesting to consider a distributed environment where clusters of queries may be formed and evaluated at different nodes of the distributed system. Another interesting issue is to consider the case when queries come in as a stream and to devise a strategy for clustering a stream of queries.

ACKNOWLEDGMENT: This work is supported in part by: NSFC $(60496322,60496327)$ and NSFHEE

(2004305). The authors would also like to express their gratitude to the anonymous reviewers for providing some very helpful suggestions.

\section{REFERENCES}

[1] H. Andrade, T. Kurç, A. Sussman, J. Saltz. Optimizing the Execution of Multiple Data Analysis Queries on Parallel and Distributed Environments. IEEE Trans. Parallel Distrib. Syst. 15(6) (2004) 520 - 532. 
[2] W. Balke, W. Nejdl, W. Siberski, U. Thaden. Progressive Distributed Top-k Retrieval in Peer-to-Peer Networks, in: Proceedings of the 21st International Conference on Data Engineering (ICDE'05), 2005, 174 185.

[3] H. Bast, D. Majumdar, R. Schenkel, M. Theobald, G. Weikum. IO-Top-k: Index-access Optimized Top-k Query Processing, in: Proceedings of 29th International Conference on Very Large Data Bases (VLDB'06), 2006, 475 - 486.

[4] S. Berchtold, C. Böhm, D. A. Keim, H.-P. Kriege, X. Xu. Optimal Multidimensional Query Processing Using Tree Striping, in: Proceedings of the Second International Conference on Data Warehousing and Knowledge Discovery (DaWaK'00), 2000, 244 - 257.

[5] N. Bruno, S. Chaudhuri, L. Gravano. Top-k Selection Queries over Relational Databases: Mapping Strategies and Performance Evaluation. ACM Transactions on Database Systems 27 (2) (2002) 153 -187.

[6] M. Carey, D. Kossmann. On saying "Enough Already!” in SQL, in: Proceedings ACM International Conference on Management of Data (SIGMOD '97), 1997, 219-230.

[7] M. Carey, D. Kossmann. Reducing the braking distance of an SQL query engine, in: Proceedings of 24th International Conference on Very Large Data Bases (VLDB '98), 1998, 158-169.

[8] K. Chakrabarti, M. Ortega-Binderberger, S. Mehrotra, K. Porkaew. Evaluating Refined Queries in Top-k Retrieval Systems. IEEE Trans. Knowl. Data Eng. 16(2) (2004) 256-270.

[9] Y.-C. Chang, L. D. Bergman, V. Castelli, C.-S.Li, M.-L. Lo, J. R. Smith. The Onion Technique: Indexing for Linear Optimization Queries, in: Proceedings ACM International Conference on Management of Data (SIGMOD '00), 2000, 391-402.

[10] S. Chaudhuri, L. Gravano, and A. Marian. Optimizing top-k selection queries over multimedia repositories. IEEE Trans. Knowl. Data Eng. 16(8) (2004) 992-1009.

[11] C. Chen, Y. Ling. A sampling-based estimator for top-k selection query, in: Proceedings of the 18th International Conference on Data Engineering (ICDE'02), 2002, 617-627.

[12] G. Das, D. Gunopulos, N. Koudas. Answering Top-k Queries Using Views, in: Proceedings of 29th International Conference on Very Large Data Bases (VLDB'06), 2006, 451 - 462.

[13] D. Donjerkovic, R. Ramakrishnan. Probabilistic optimization of top N queries, in: Proceedings of 25th International Conference on Very Large Data Bases (VLDB'99), 1999, 411-422.

[14] F. Fabret, H. A. Jacobsen, F. Llirbat, J. Pereira, K. Ross, D. Shasha. Filtering Algorithms and Implementation for Very Fast Publish/Subscribe systems, SIGMOD Conference 2001, 115-126.

[15] R. Fagin, A. Lotem, M. Naor. Optimal aggregation algorithms for middleware, in: Proceedings of the Twentieth ACM Symposium on Principles of Database Systems (PODS '01), 2001, 102 - 113.

[16] R. Fenk, V. Markl, R. Bayer. Improving multidimensional range queries of non-rectangular volumes specified by a query box set, in: Proceedings of Databases, Web and Cooperative Systems (DWACOS) 1999.

[17] D. Habich, W. Lehner, A. Hinneburg. Optimizing multiple top-K queries over joins, in: Proceedings of the 17th international conference on Scientific and statistical database management, 2005,195 - 204. 
[18] V. Hristidis, N. Koudas, Y. Papakonstantinou. PREFER: A System for the Efficient Execution of Multiparametric Ranked Queries, in: Proceedings of the 2001 ACM International Conference on Management of Data (SIGMOD ’01), 2001, 259-270.

[19] V. Hristidis and Y. Papakonstantinou. Algorithms and applications for answering ranked queries using ranked views. VLDB Journal, 13(1) (2004) 49-70.

[20] I. Ilyas, W. Aref, A. Elmagarmid. Supporting top-k join queries in relational databases. VLDB J. 13(3) (2004) 207-221.

[21] I. Ilyas, W. Aref, A. Elmagarmid. Joining ranked inputs in practice, in: Proceedings of 28th International Conference on Very Large Data Bases (VLDB'02), 2002, 950-961.

[22] I. Ilyas, R. Shah, W. Aref, J. Vitter, A. Elmagarmid. Rank-aware query optimization, in: Proceedings ACM International Conference on Management of Data (SIGMOD’04), 2004, 203-214.

[23] J. Lee, D. Kim, C. Chung. Multi-dimensional Selectivity Estimation Using Compressed Histogram Information, in: Proceedings ACM International Conference on Management of Data, (SIGMOD'99), 1999, 205-214.

[24] C. Li, K. Chang, I. Ilyas, S. Song. RankSQL, Query Algebra and Optimization for Relational Top-k Queries, in: Proceedings ACM International Conference on Management of Data (SIGMOD’05), 2005, 131-142.

[25] A. Marian, N. Bruno, L. Gravano. Evaluating top-k queries over web-accessible databases, ACM Trans. Database Syst. 29(2) (2004) 319-362.

[26] W. Meng, C. Yu, W. Wang, N. Rishe. Performance Analysis of Three Text-Join Algorithms, IEEE Trans. Knowl. Data Eng. 10(3) (1998) 477-492.

[27] S. Michel, P. Triantafillou, G. Weikum. KLEE: A Framework for Distributed Top-k Query Algorithms, in: Proceedings of the 31st International Conference on Very Large Data Bases (VLDB'05), 2005, 637-648.

[28] A. Motro. VAGUE: A user interface to relational databases that permits vague queries. ACM Trans. Office Inf. Syst. 6 (3) (1988), 187-214.

[29] K. Mouratidis, S. Bakiras, D. Papadias. Continuous Monitoring of Top-k Queries over Sliding Windows. in: Proceedings ACM International Conference on Management of Data (SIGMOD’06), 2006, 635-646.

[30] P. O’Neil and E. O’Neil. Database: Principles, Programming, and Performance, 2nd edition, Morgan Kaufmann Publishers, 2001.

[31] D. Papadias, Q. Shen, Y.Tao, K. Mouratidis. Group Nearest Neighbor Queries, in: Proceedings of the 20th International Conference on Data Engineering (ICDE’04), 2004, 301-312.

[32] T. Sellis, Multiple-Query Optimization. ACM Trans. Database Syst. 13(1)( 1988) 23-52.

[33] T. Sellis, S. Ghosh, On the Multiple-Query Optimization Problem, IEEE Trans. Knowl. Data Eng. 2(2) (1990) 262-266.

[34] A. Silberschatz, H. F. Korth, S. Sudarshan. Database System Concepts, 4th Edition. McGraw-Hill, 2002.

[35] Ion Stoica. A Time-Optimal Multiple-Query Nearest-Neighbor Algorithm on Meshes with Multiple Broadcasting, International Journal of Pattern Recognition and Artificial Intelligence 9(4) (1995) 663-677.

[36] I. Taksa. Predicting the Cumulative Effect of Multiple Query Formulations, International Symposium on Information Technology: Coding and Computing ( ITCC(2)’05), 2005, 491-496. 
[37] M. Theobald, G. Weikum, R. Schenkel. Top-k Query Evaluation with Probabilistic Guarantees, in: Proceedings of the Thirtieth International Conference on Very Large Data Bases (VLDB’04), 2004, 648-659.

[38] M. Wojciechowski, M. Zakrzewicz. On Multiple Query Optimization in Data Mining, in: Proc. Advances in Knowledge Discovery and Data Mining, 9th Pacific-Asia Conference (PAKDD’05), Lecture Notes in Computer Science, 3518 (Springer 2005), 696-701.

[39] D. Xin, J. Han, H. Cheng, X. Li. Answering Top-k Queries with Multi-Dimensional Selections: The Ranking Cube Approach, in: Proceedings of 29th International Conference on Very Large Data Bases (VLDB’06), 2006, $463-474$.

[40] K. Yi, H. Yu, J. Yang, G. Xia, and Y. Chen. Efficient maintenance of materialized top-k views, in: Proceedings of the 19th International Conference on Data Engineering (ICDE'03), 2003, 189-200.

[41] C. Yu, G. Philip, W. Meng. Distributed Top-N Query Processing with Possibly Uncooperative Local Systems, in: Proceedings of 29th International Conference on Very Large Data Bases (VLDB'03), 2003, 117-128.

[42] C. Yu, P. Sharma, W. Meng, Y. Qin. Database selection for processing k nearest neighbors queries in distributed environments, in: ACM/IEEE Joint Conference on Digital Libraries (JCDL), 2001, 215-222.

[43] L. Zhu, W. Meng. Learning-Based Top-N Selection Query Evaluation over Relational Databases, in: Advances in Web-Age Information Management: 5th International Conference (WAIM'04), Lecture Notes in Computer Science, 3129 (Springer 2004), 197-207.

[44] M. Zhu, D. Papadias, J. Zhang, D. Lee. Top-k Spatial Joins, IEEE Trans. Knowl. Data Eng. 17(4) (2005), 567579. 